\title{
Voxel-wise Optimization of Pseudo Voigt Profile (VOPVP) for Z-spectra fitting in chemical exchange saturation transfer (CEST) MRI
}

\author{
Lihong Zhang, Yingcheng Zhao, Yanrong Chen, Chongxue Bie, Yuhua Liang, Xiaowei He, Xiaolei Song \\ School of Information Science and Technology, Northwest University, Xi'an 710127, China
}

Correspondence to: Xiaolei Song. School of Information Science and Technology, Northwest University, Xi'an 710127, China. Email: xlsong8@163.com.

Background: Chemical exchange saturation transfer (CEST) MRI is a promising approach for detecting biochemical alterations in cancers and neurological diseases, but the quantification can be challenging. Among numerous quantification methods, Lorentzian difference (LD) is relatively simple and widely used, which employs Lorentzian line-shape as a reference to describe the direct saturation (DS) of water and takes account of difference against experimental CEST spectra data. However, LD often overestimates CEST and nuclear overhauser enhancement (NOE) effects. Specifically, for fast-exchanging CEST species require higher saturation power $\left(\mathrm{B}_{1_{1} \text { sat }}\right)$ or in the presence of strong magnetization transfer (MT) contrast, Z-spectrum appears more like a Gaussian line-shape rather than a Lorentzian line-shape.

Methods: To improve the conventional LD analysis, the present study developed and validated a novel fitting algorithm through a linear combination of Gaussian and Lorentzian function as the reference spectra, namely, Voxel-wise Optimization of Pseudo Voigt Profile (VOPVP). The experimental Z-spectra were pre-fitted with Gaussian and Lorentzian method independently, in order to determine Lorentzian proportionality coefficient (a). To further compensate for the line-shape changes under different $B_{1_{\text {_sat }}}$ 's, a $B_{1^{-}}$dependent adjustment was applied to the experimental $Z$-spectra $\left(Z_{\text {exp }}\right)$ according to the prior knowledge learned from 5-pool Bloch equation-based simulations at a range of $B_{1_{-} \text {sat }}$ 's. Then, the obtained $Z$-spectra $\left(Z_{-B 1 a d j}\right)$ was fitted by the previously defined VOPVP function. Considering the asymmetric component of MT, the positive- and negative-side of Z-spectra were fitted separately, while the middle part (-0.6 to $0.6 \mathrm{ppm}$, consisted primarily of DS) was fitted using Lorentzian function. Finally, the difference between $Z_{\text {_VOPVP }}$ and $Z_{\text {exp }}$ was defined as the CEST and NOE contrast. To validate our VOPVP method, an extensive simulation of CEST Z-spectra was performed using 5-pool model and 6-pool model with greater MT component.

Results: In comparison with LD approach, VOPVP exhibited lower sum of squares due to error (SSE) and

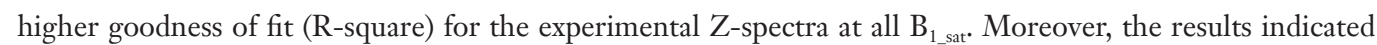
that VOPVP fitting improved the overestimated contributions from amide proton transfer (APT) and NOE

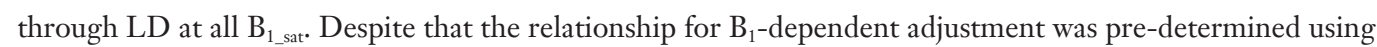
a single 5-pool model, the VOPVP fittings obtained accurate quantification for multiple 6-pool models with a range of $T_{1}$ w's and $T_{2}$ w's. The robustness of VOPVP fitting was also proved by simulations using $3 \mathrm{~T}$ parameters. Furthermore, we assessed VOPVP in vivo in a glioblastoma-bearing mouse. Compared to LD maps, VOPVP quantification maps displayed higher contrast-to-noise ratio between tumor and normal contralateral tissue for APT, glutamate and nuclear overhauser effect (NOE), when $\mathrm{B}_{1 \_ \text {sat }}>1 \mu \mathrm{T}$.

Conclusions: As an improvement of LD method, VOPVP fitting can serve as a simple, robust and more accurate approach for quantifying CEST and NOE contrast.

Keywords: Chemical exchange saturation transfer (CEST); Lorentzian fit; amide proton transfer (APT); nuclear overhauser enhancement (NOE); Pseudo Voigt profile 
Submitted Jul 16, 2019. Accepted for publication Sep 29, 2019.

doi: 10.21037/qims.2019.10.01

View this article at: http://dx.doi.org/10.21037/qims.2019.10.01

\section{Introduction}

Chemical exchange saturation transfer (CEST) is a magnetic resonance imaging (MRI) technique for detecting lowconcentration metabolites and molecules with exchangeable protons at specific resonance frequencies, which can be labeled by a saturation pulse allowing signal transfer to water pool for detection (1-3). CEST MRI $(1,4)$ has been applied to detect exogenous or endogenous amine (5), amide (6), creatine (7), glucose $(8,9)$, glutamate (10), glycogen (11) and glycosaminoglycan (12). Additionally, CEST technique can probe the micro-environment of tissue, including temperature (13) and $\mathrm{pH}(14,15)$. These unique capabilities of CEST MRI make it a promising target for in vivo imaging applications such as the diagnosis of stroke (16-20) as well as detection and grading of tumors (9,21-31).

CEST MRI often involves a series of images with saturation pulse sweeping over a range of frequency offsets, with the signal changes along the extracted offset, also termed as Z-spectra (32). The most commonly used CEST quantification method, namely, MT ratio asymmetry $\left(\mathrm{MTR}_{\text {asym }}\right)$ analysis (33), takes the difference between two CEST images at opposite frequency offsets $(+/-\Delta \omega)$ as

$$
M T R_{\text {asym }}=\frac{S_{\text {sat }}(-\Delta \omega)-S_{\text {sat }}(\Delta \omega)}{S_{0}}
$$

where $S_{\text {sat }}(-\Delta \omega)$ and $S_{\text {sat }}(\Delta \omega)$ are signals with saturated $R F$ irradiation, which applied at the reference frequency and labeled proton frequency; $S_{0}$ is the reference signal without RF irradiation. MTR $_{\text {asym }}$ is simple and easy to be calculated, which has been shown to correlate with tumor grade in case of amide proton transfer (APT) (34-36). However, $\mathrm{MTR}_{\text {asym }}$ is susceptible to several types of contamination, including $\mathrm{B}_{0}$ inhomogeneity (37), direct saturation (DS) and semisolid macromolecular magnetization transfer (MT). Moreover, it is incapable to separate nuclear overhauser effect (NOE) at resonance frequencies up field of water based on CEST contrast.

To further improve CEST specificity and its signal quantification, $\mathrm{Z}$-spectra fitting has been employed to distinguish the contributions from multiple origins (38). Theoretically, the enhancement of CEST through Z-spectra is dependent on the pool size, exchange rate and relaxation time $(25,34)$, as demonstrated by Bloch-McConnell equations. However, the complex Bloch fitting strongly relies on the initialization and boundaries of fitting parameters (39). According to the shape of $Z$-spectra, other quantification methods have been proposed, including multiple-pool Lorentzian fitting $(7,24,38-40)$ and Lorentzian difference (LD) analysis (18,40-42). Multiple-pool Lorentzian fits each 'dip' in Z-spectra using a Lorentzian shape, in which the reference signals can be obtained by setting the fitted amplitude of the target CEST or NOE pools to zero. However, multiple-pool Lorentzian fitting requires the Z-spectra to be collected at a sufficient sampling frequency, and thus such method is time consuming. In addition, it has different fitting parameters, and is sensitive to the signalto-noise ratio of $\mathrm{Z}$-spectra. Besides, the $\mathrm{LD}$ method is a simplified method that employs a single Lorentzian line as a reference to describe the DS and takes account of difference against experimental data for quantifying CEST and NOE signal. LD can be an easy and robust quantification method, especially at low $\mathrm{B}_{1 \_ \text {sat }}(\leq 1 \mu \mathrm{T}$ at 9.4 Tesla) (40), which has been initially validated in patients with stroke (18,40-42). However, LD analysis possesses the disadvantage of overestimating contributions from CEST and NOE effects (40). In particular, for fast-exchanging species that require higher $B_{1 \_ \text {sat }}$ or for tissue with a strong MT contrast, the Z-spectra exhibit non-Lorentzian line-shape (40) making the results of LD analysis invalid. More importantly, the Z-spectrum appears more like a Gaussian line-shape, rather than a Lorentzian line-shape, when $\mathrm{B}_{1 \text { _sat }}$ is higher or in the presence of strong MT contrast $(20,43)$.

During NMR spectroscopy analysis, Voigt spectra line profile is often defined by the convolution of Lorentzian and Gaussian terms (44-46). As an excellent approximation to Voigt profile, Pseudo Voigt profile is best defined as the weighted sum of Gaussian and Lorentzian (47). In this study, a Voxel-wise Optimization Pseudo Voigt Profile (VOPVP) fitting algorithm was developed to improve the reliability of in vivo CEST MRI quantification. To further compensate for the different levels of MT and DS, a $\mathrm{B}_{1 \text { _sat }}$-dependent optimization was adopted into the VOPVP fitting based on the 5-pool Bloch simulations under different $B_{1 \_ \text {sat }}$. To evaluate the performance of VOPVP 


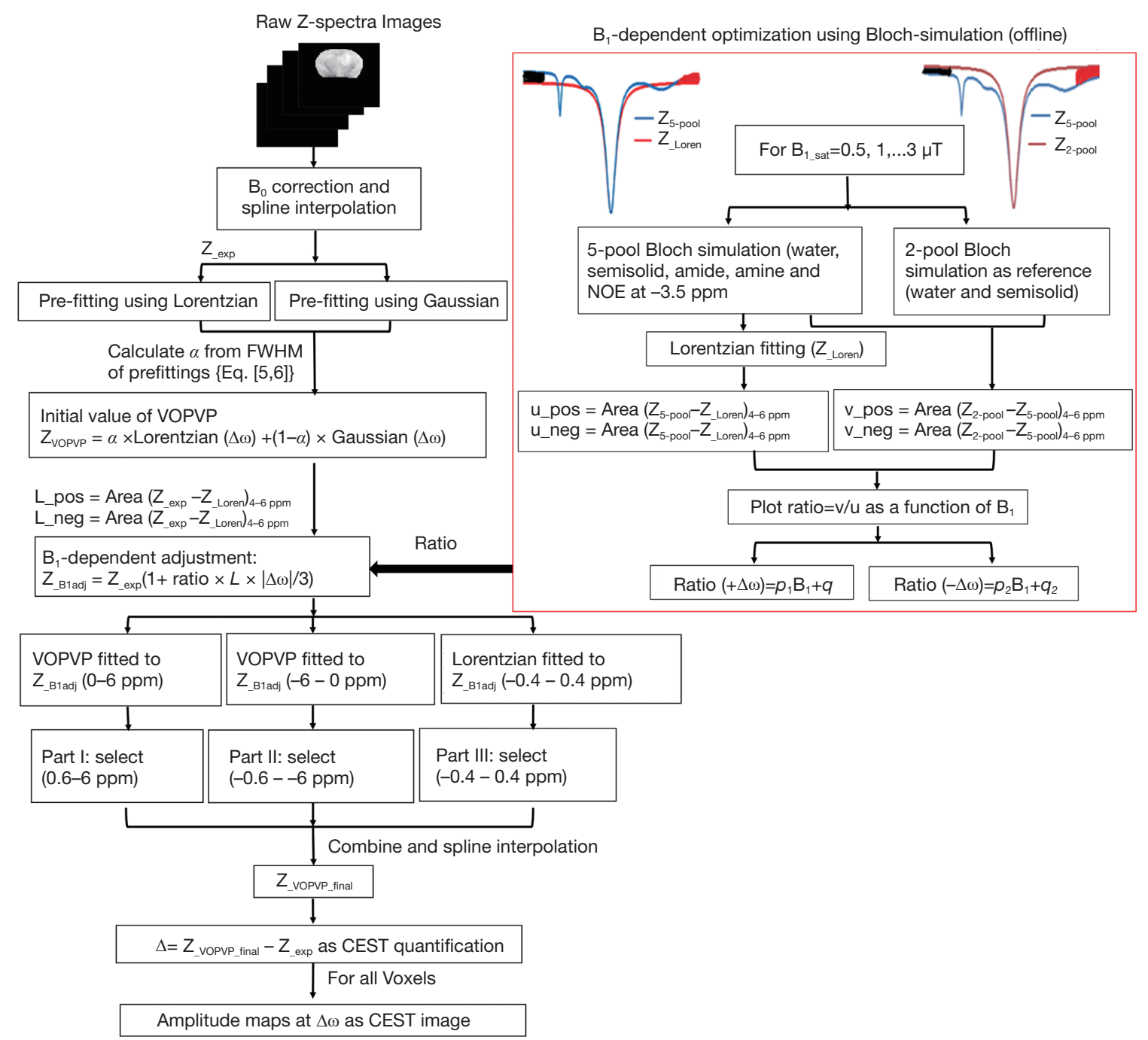

Figure 1 Flow chart of data processing steps of Voxel-wise Optimization Pseudo Voigt Profile (VOPVP) fitting approach.

fitting, an extensive Bloch-simulations was performed using previously published parameters (i.e., 5-pool model and 6-pool model with greater MT contributions) at various $\mathrm{B}_{1 \_ \text {sat }}$. The conventional LD and an analytical standard were also assessed and compared. Finally, an initial validation for in vivo application was carried out using a brain tumorbearing mouse.

\section{Methods}

\section{Theoretical concepts}

The detailed flow of our VOPVP method is illustrated in Figure 1. The proposed fitted function, or termed as Pseudo
Voigt profile (47), was expressed by a weighted sum of Gaussian (G) and Lorentzian (L):

$$
V(\Delta \omega) \approx \alpha \times L(\Delta \omega)+(1-\alpha) G(\Delta \omega)
$$

where $\alpha$ and $1-\alpha$ denote the proportionality coefficients of Lorentzian and Gaussian functions, respectively. The model function of Gaussian fitting can be described by

$$
\mathrm{G}(\Delta \omega)=\frac{1}{\sqrt{2 \pi} \sigma} e^{\frac{-\left(\omega_{1}-\omega\right)^{2}}{2(\sigma)^{2}}}
$$

where $\omega_{1}$ is the frequency offset from the water resonance, while $\omega$ is the frequency offset of the CEST peak for the proton pool.

The model function of Lorentzian fitting can be given by 

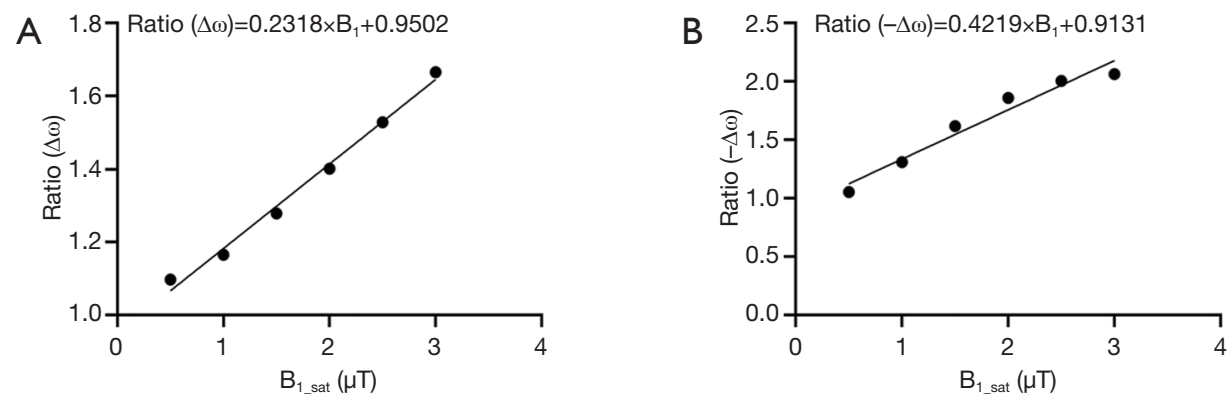

Figure 2 Relationship between (A) Ratio $(\Delta \omega)$, (B) Ratio $(-\Delta \omega)$, and $B_{1_{-} \text {sat }}$.

$$
\mathrm{L}(\Delta \omega)=\frac{A}{\pi\left[1+\frac{\omega_{1}-\omega}{\sigma}\right]^{2}}
$$

where $\omega_{1}$ is the frequency offset from the water resonance, whereas A, $\omega$ and $\sigma$ are the amplitude, frequency offset and linewidth of the CEST peak for the proton pool, respectively.

Thompson et al. (45) have proposed the following expression for the pseudo-Voigt approximation for the convolution of both Gaussian and Lorentzian functions, as presented by the full width at half maximum (FWHM) values of Gaussian and Lorentzian:

$$
a=1.36603\left(\frac{\Gamma_{\mathrm{L}}}{\Gamma}\right)-0.47719\left(\frac{\Gamma_{\mathrm{L}}}{\Gamma}\right)^{2}+0.11116\left(\frac{\Gamma_{\mathrm{L}}}{\Gamma}\right)^{3}
$$

where

$$
\begin{aligned}
\Gamma= & \left(\Gamma_{G}^{5}+2.69269 \Gamma_{G}^{4} \Gamma_{L}+2.42843 \Gamma_{G}^{3} \Gamma_{L}^{2}+4.47163 \Gamma_{G}^{2} \Gamma_{L}^{3}\right. \\
& \left.+0.07842 \Gamma_{G} \Gamma_{L}^{4}+\Gamma_{L}^{5}\right)^{1 / 5}
\end{aligned}
$$

where $\Gamma_{G}$ and $\Gamma_{L}$ are the FWHM of Gaussian and Lorentzian, respectively.

\section{VOPVP fitting}

Figure 1 shows the flow chart of the proposed VOPVP fitting algorithm. Firstly, for the raw Z-spectra data, WASSR was performed to correct $B_{0}$ inhomogeneity.

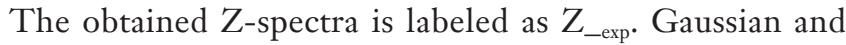
Lorentzian model pre-fitting was performed for $Z_{\text {_exp }}$ in order to achieve the Lorentzian proportionality coefficient (a), based on the FWHM of Gaussian and Lorentzian derived from Eqs. [5] and [6], respectively. Subsequently, the area values of $Z_{- \text {loren }}$ under $Z_{\text {-exp }}$ between 4 and 6 ppm and between -4 and -6 ppm are named as $L_{-}$pos and L_neg, respectively.
To further compensate for the line-shape changes under different $B_{1 \_ \text {sat }}$ 's, a $B_{1}$-dependent adjustment was applied to the experimental $Z$-spectra $\left(Z_{-\exp }\right)$ according to the prior knowledge learned from 5-pool Bloch equation-based simulations at a range of $B_{1_{-} \text {sat }}$ 's. Simulated $Z$-spectra $\left(Z_{5 \text {-pool }}\right)$ of brain tissue with $\mathrm{B}_{0}=9.4 \mathrm{~T}$ were obtained through 5 -pool Bloch equation-based simulations (48), for a range of $B_{1_{\_} \text {sat }}$ $(0.5,1,1.5,2,2.5$ and $3 \mu \mathrm{T})$. Accurate reference spectra describing DS and MT were determined using a 2-pool model (water and semisolid), with the residual with $Z_{5 \text {-pool }}$ for CEST quantification. The Lorentzian fitting was also performed. The residual areas with regarding to $Z_{5 \text {-pool }}$, were calculated for Lorentzian fitting $\left[u_{-}\right.$pos (4 to $6 \mathrm{ppm}$ ) and $u_{-}$ neg ( -4 to $-6 \mathrm{ppm})]$, and also for the 2-pool model [v_pos (4 to $6 \mathrm{ppm})$ and $v_{-}$neg $(-4$ to $\left.-6 \mathrm{ppm})\right]$. Then, for each $\mathrm{B}_{1_{\text {_sat }} \text {, }}$, the two ratios of $\Delta \omega$ and $-\Delta \omega$ were calculated as follows: (A) Ratio $(\Delta \omega)=v \_p o s / u \_p o s$. (B) Ratio $(-\Delta \omega)=v \_n e g / u \_n e g$. Figure 2 plotted Ratio $(\Delta \omega)$ and Ratio $(-\Delta \omega)$ as a function of $B_{1 \_s a t}$. As seen, when $B_{1 \_ \text {sat }}<1 \mu \mathrm{T}$, ratio values are close to 1 , indicating accurate quantification of LD. However, when $\mathrm{B}_{1_{\text {sat }}} \geq 1 \mu \mathrm{T}$ LD become inaccurate with ratio $>1$. For both Ratio $(\Delta \omega)$ and Ratio $(-\Delta \omega)$, a linear relationship with $\mathrm{B}_{1_{\_} \text {sat }}$ can be determined, providing the prior knowledge of fitting errors. Here, before starting VOPVP optimization, a $\mathrm{B}_{1}$-dependent adjustment was applied to $Z_{-\exp }$, resulting in $Z_{\text {B1adj }}=Z_{\text {exp }}(1+$ Ratio $\times L \times|\Delta \omega| / 3)$. By multiplying $Z_{\text {_exp }}$ with the Ratio values at the applied $B_{1_{1} \text { sat }}$, the adjusted spectra $\left(Z_{B_{B 1 a d j}}\right)$ can compensate for the difference between LD and accurate quantification.

Then, with the initial value of VOPVP fitting defined by Eqs. [2-4], the fitting to $Z_{- \text {B1adj }}$ was divided into three parts. Part I, VOPVP function was used to fit the $Z_{-B 1 \text { adj }}$ between 0 and 6 ppm. Part II, VOPVP function was used to fit the $Z_{-B \text { Badj }}$ between -6 and 0 ppm. Part III, Lorentzian was employed to fit the $Z_{\text {B Badj }}$ between -0.4 and $0.4 \mathrm{ppm}$. The final fitting results integrated the fitted data from part 
Table 1 Parameters for the Bloch equation-based simulations (9.4T)

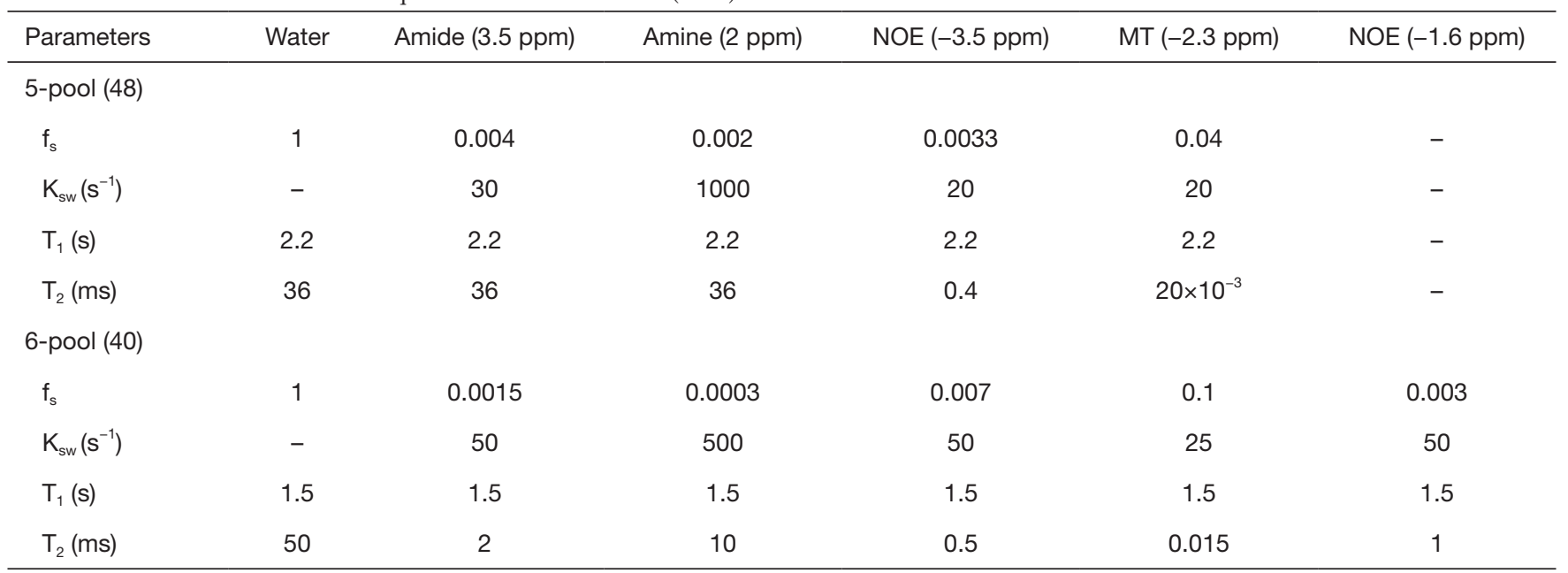

NOE, nuclear overhauser enhancement; MT, magnetization transfer.

I (0.6 to $6 \mathrm{ppm})$, part II (-6 to $-0.6 \mathrm{ppm})$ and part III $(-0.4$ to $0.4 \mathrm{ppm})$. Then, Spline interpolation was used to assess the final fitting data, namely, $Z_{\text {_vopvp_final }}$. For all Voxels, the difference between $Z_{\text {VVOPVP_final }_{\text {V }}}$ and $Z_{\text {_exp }}$ was calculated to obtain the amplitude of maps at $\Delta \omega$. DS effects in weak range at both sides of $0 \mathrm{ppm}$ with the line-shape similar to Lorentzian were fitted into the part III. The Z-spectra was far from 0 ppm and might contain MT components, which made Pseudo Voigt Profile an ideal fitting method. Moreover, there was no exchangeable proton within $0.6 \mathrm{ppm}$, according to the exchangeable proton chemical shifts for various diamagnetic agents (49) ranging from 0 to $7 \mathrm{ppm}$. Therefore, only the accuracy of fitting within \pm 6 ppm was considered.

\section{LD fitting}

In addition, Lorentzian difference (LD) fitting $(40,50)$ was carried out during the simulation. In this method, Lorentzian fitting was performed to evaluate the values of Z-spectra ( -10 to $-6.25 \mathrm{ppm},-1.7$ to $1.7 \mathrm{ppm}$ and 6.25 to $10 \mathrm{ppm}$ at $9.4 \mathrm{~T})$, while spline interpolation was used to complete the entire fitting process. The fitted spectra were adopted as reference signals, representing the DS and semisolid MT effects. The residual spectra of CEST (40) were formed by subtracting the measured Z-spectra from the fitted spectra.

For in vivo experiments, the offsets greater than $\pm 6 \mathrm{ppm}$ were not collected due to the restriction of scanning time. To improve LD performance, a compensate strategy similar to previous research (18) was employed, where the acquired Z-spectra were normalized by $\mathrm{S}_{\text {sat }}(-6 \mathrm{ppm})$ of the lower $\mathrm{B}_{1-\text { sat, }}$, and then fitted by Lorentzian fitting.

\section{Simulation}

To assess the performance of the proposed VOPVP method, Bloch equation-based simulations were carried out using both 5-pool exchange model (free water, amide, amine, MT and NOE at -3.5 ppm) (51) and 6-pool model (free water, amide, amine, MT, NOE at $-3.5 \mathrm{ppm}$ and NOE at $-1.6 \mathrm{ppm})$ (40). Simulation parameters for rodent brain tissue at 9.4 Tesla are listed in Table 1. Specifically, the 6-pool model contained more MT and NOE components but less amides and amines in comparison with 5-pool model $(40,48)$. To further evaluate the robustness of our method, simulations were performed at six different $\mathrm{B}_{1 \_ \text {sat }}$ levels, i.e., 0.5, 1, 1.5, 2, 2.5 and $3 \mu \mathrm{T}$.

To assess the robustness of our VOPVP method, we further performed simulation for a range of $\mathrm{T}_{1} \mathrm{w}(0.5$ to $2.5 \mathrm{~s}$ ) and $\mathrm{T}_{2} \mathrm{w}(25$ to $125 \mathrm{~ms})$, all using the same ratio values. To prove the feasibility at clinical field strengths, the ratios were calculated (Figure S1) using the parameters at $3 \mathrm{~T}$ for rodent brain tissue (Table S1). Then the VOPVP fittings were performed and compared, using either ratios calculated from $3 \mathrm{~T}$, or those from 9.4T.

\section{In vivo experiment}

Animal experiments were performed in accordance with the guidelines of Johns Hopkins University Animal Care and 
Use Committee guidelines. Balb/c NOD/SCID mouse were xenografted intracranially with 100,000 human glioblastoma cells to striatum of the brain $(52,53)$ with MR imaging performed 6-week post-injection. In vivo MRI experiments were conducted on an $11.7 \mathrm{~T}$ horizontal bore scanner (Bruker Biospec, Germany) using a transmit-receiver volume coil (23 $\mathrm{mm}$ diameter). CEST MRI images were acquired using a continuous wave pre-saturation pulse (Tsat $=2,500 \mathrm{~ms}$ ), followed by a rapid acquisition with relaxation enhancement (RARE) readout (RARE factor $=12$ ). $B_{0}$ inhomogeneity was then corrected using WASSR (54). The Z-spectra were acquired from -6 to $6 \mathrm{ppm}$ with an interval of $0.25 \mathrm{ppm}$ at three $\mathrm{B}_{1_{-} \text {sat }}$ of $0.8,1.2$ and $2.4 \mu \mathrm{T}$. The other parameters were as follows: TR/TE $=5,500 \mathrm{~ms} /$ $11 \mathrm{~ms}$, slice thickness $=1 \mathrm{~mm}, \mathrm{FOV}=17 \times 14 \mathrm{~mm}^{2}$ and matrix size $=96 \times 64$.

\section{Evaluation criteria}

The obtained data were evaluated by four parameters: (I) the sum of squares due to error (SSE), (II) coefficient of (R-square) determination (39), (III) simple analytic solution to the apparent exchanged-dependent relaxation (AREX) $(20,23,51,55,56)$, and (IV) contrast-to-noise ratio (CNR) for in vivo experiments (39). The AREX was further defined as follows:

To evaluate the accuracy of CEST quantification methods in numerical simulations, we used $R_{e x}^{C E S T}(\Delta \omega)$ $(20,40,55,57)$ as a gold standard, which is independent of non-specific tissue parameters such as $\mathrm{T}_{1 \mathrm{w}}$, DS and semisolid MT effects:

$R_{e x}^{C E S T}(\Delta \omega)=\frac{f_{s} k_{s w} \omega_{1}^{2}}{\omega_{1}^{2}+\left(\mathrm{R}_{2 s}+k_{s w}\right) k_{s w}+(\Delta \omega-\Delta)^{2} k_{s w} /\left(\mathrm{R}_{2 s}+k_{s w}\right)}[7]$

where $f_{s}$ is solute concentration, $k_{s w}$ is solute-water exchange rate, $R_{2 s}$ is solute transverse relaxation and $\omega_{1}$ is irradiation power. In comparison with $R_{e x}^{C E S T}(\Delta \omega)$, AREX, the inverse metric of the Z-spectra fitted by LD and VOPVP, was defined as below $(40,55)$ :

$$
\operatorname{AREX}(\Delta \omega)=\left(\frac{1}{Z_{\text {lab }}(\Delta \omega)}-\frac{1}{Z_{r e f}(\Delta \omega)}\right) R_{\text {lobs }}\left(1+f_{c}\right)
$$

where $f_{c}$ is semi-solid MT pool size ratio.

\section{Results}

\section{Simulations}

Figure 3 displays the simulated Z-spectra, VOPVP fitting and LD fitting of $\mathrm{B}_{1_{\text {sat }}}$ at $0.5,1,1.5,2,2.5$ and $3 \mu \mathrm{T}$, apart from the difference between VOPVP and the simulated data. For the simulated data through 5-pool model, the difference spectra generated by VOPVP and Lorentzian fitting could reveal the peaks arising from amide proton at $3.5 \mathrm{ppm}$, amine protons at $2 \mathrm{ppm}$ and $\mathrm{NOE}$ at $-3.5 \mathrm{ppm}$ (NOE at -1.6 ppm for 6-pool only). However, LD overestimated the amide, amine CEST signals when $\mathrm{B}_{1 \_ \text {sat }}$ $=1,1.5$ and $2 \mu \mathrm{T}$, while underestimated $\mathrm{NOE}$ when $\mathrm{B}_{1_{\text {_sat }}}$ $=2.5$ and $3 \mu \mathrm{T}$. In contrast, for all the saturation powers, our VOPVP method could provide a closer fitting to the nonspecific parts of Z-spectra (-6 to $-5 \mathrm{ppm},-1.5$ to $1.5 \mathrm{ppm}$ and 4 to $6 \mathrm{ppm}$ ), as quantitatively indicated by both SSE and R-square (Table 2). As aforementioned, the 6-pool model may contain more MT and NOE components but less amides and amines than the 5-pool model. Our results showed the SSE of 6-pool increased by up to $100 \%$ from 5 -pool model (Table 2$)\left(\mathrm{B}_{1 \_ \text {sat }}=3 \mu \mathrm{T}\right)$ is not only NOE at -1.6 ppm but also the 6-pool model containing more MT components.

In addition, the AREX metrics derived from VOPVP fitting and LD fitting were further compared with $R_{e x}^{C E S T}$ analytical standard (Figure 4 and Table 3). For the 6-pool model, LD overestimated all the CEST and NOE signals. For the 5-pool model, LD overestimated amides and NOE $(3.5 \mathrm{ppm})$ at $\mathrm{B}_{1 \_ \text {sat }} \leq 2 \mu \mathrm{T}(1,1.5$ and $2 \mu \mathrm{T})$, while underestimated APT and NOE (3.5 ppm) at $3 \mu \mathrm{T}$. For both the 5-pool and the 6-pool models, the derived AREX $\mathrm{X}_{\mathrm{VOPVP}}$ spectra were significantly closer to the standard $R_{e x}^{C E S T}$ spectra, especially at $\mathrm{B}_{1 \_ \text {sat }} \geq 1 \mu \mathrm{T}(1,1.5,2,2.5$ and $3 \mu \mathrm{T})$. Table 3 lists the ratios of the peak intensities of the AREX $\mathrm{LD}_{\mathrm{LD}}$ and AREX $X_{V O P V P}$ metrics to those of $R_{e x}^{C E S T}$. Notably, for the 5 -pool model, AREX $\mathrm{VOPVP}_{\mathrm{V}}$ at amide frequency $(3.5 \mathrm{ppm})$ and NOE $(-3.5 \mathrm{ppm})$ were relatively close to $R_{e x}^{\text {CEST }}$ at all $\mathrm{B}_{1 \_ \text {sat }}$. While for the 6-pool model with a greater MT contribution, AREX $X_{\mathrm{VOPVP}}$ could estimate $R_{e x}^{\text {CEST }}$ more accurately at lower $B_{1 \_ \text {sat }}(\leq 2 \mu \mathrm{T})$, but still underestimated the APT at 2.5 and $3 \mu \mathrm{T}$; Nevertheless, for all cases, $\mathrm{AREX}_{\mathrm{VOPVP}}$ outperformed $\mathrm{AREX} \mathrm{X}_{\mathrm{LD}}$ with regard to all peaks (i.e., APT, amine and NOE).

The fitted linear function at 3T (Figure S1) suggested a slightly different slope and shift compared with those calculated at 9.4T (Figure 2). As seen, $\mathrm{AREX}_{\mathrm{VOPVP}}$ values were closer to the analytical solution $R_{e x}^{\text {CEST }}$ than AREX $\mathrm{LD}_{\mathrm{LD}}$ values, for both $\mathrm{B}_{1_{1} \text { sat }}=1 \mu \mathrm{T}$ and $\mathrm{B}_{1_{\text {sat }}}=2 \mu \mathrm{T}$ (Figure 5). As seen, the two kinds of VOPVP fitting curves are almost identical, which both resulted in accurate quantification $\left(\mathrm{AREX}_{\mathrm{VOPVP}}\right)$ compared with the analytical standard $R_{e x}^{C E S T}$ ) 
A
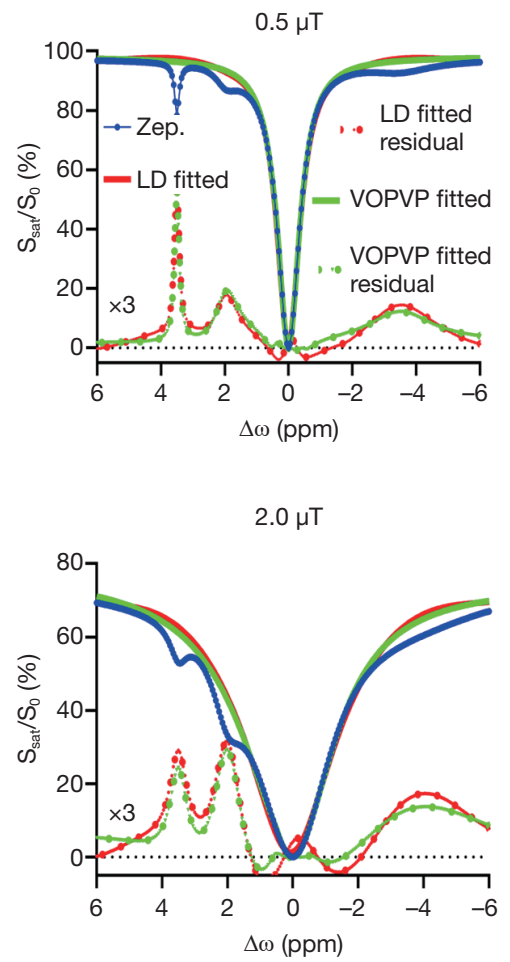

B
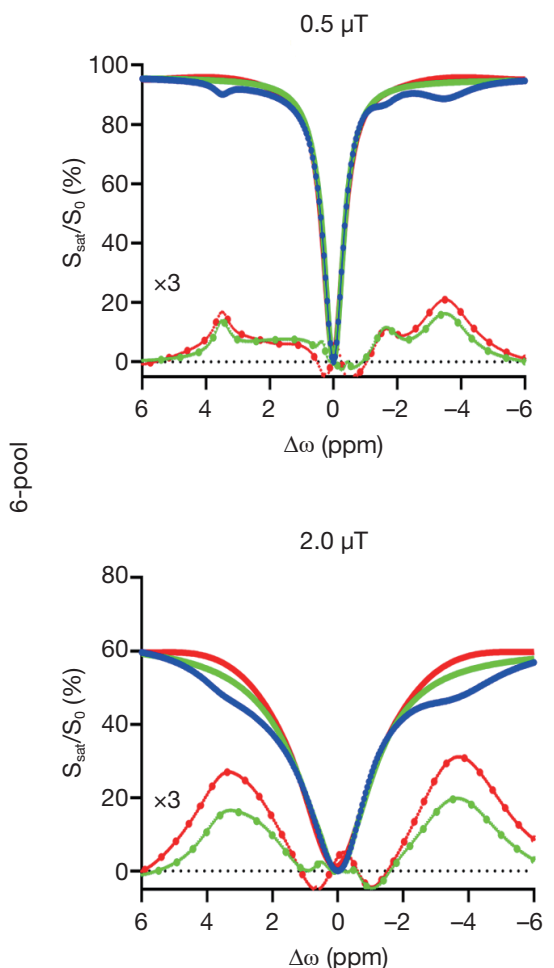

$1.0 \mu \mathrm{T}$

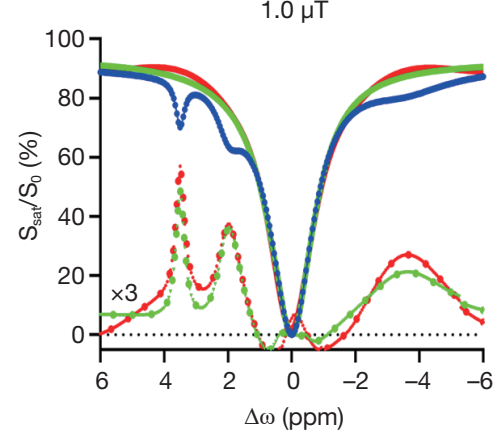

$2.5 \mu \mathrm{T}$

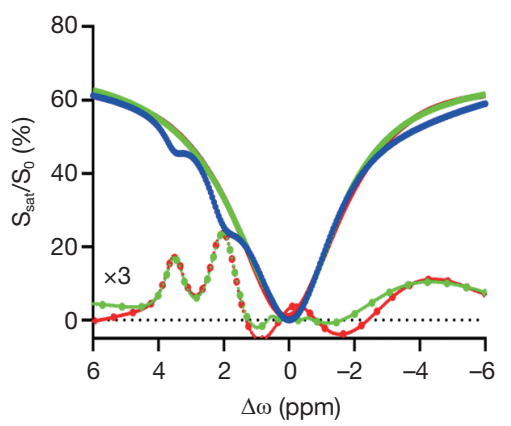

$1.0 \mu \mathrm{T}$

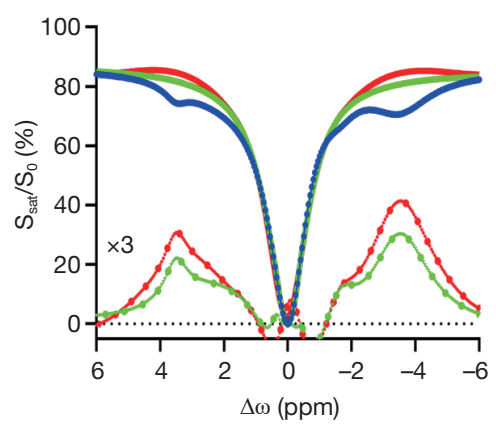

$2.5 \mu \mathrm{T}$

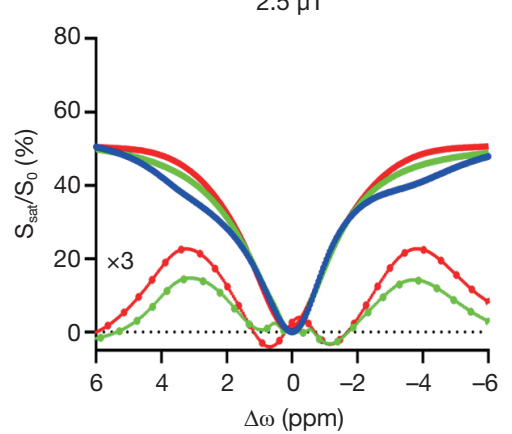

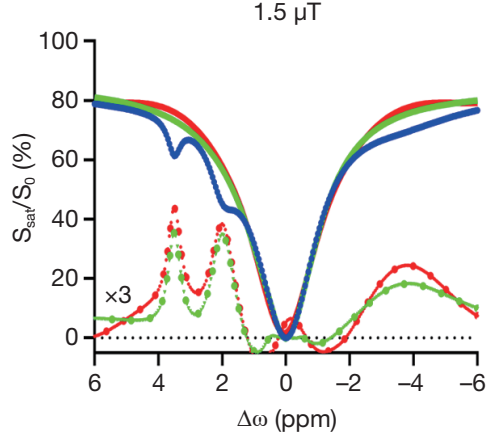

$3.0 \mu \mathrm{T}$

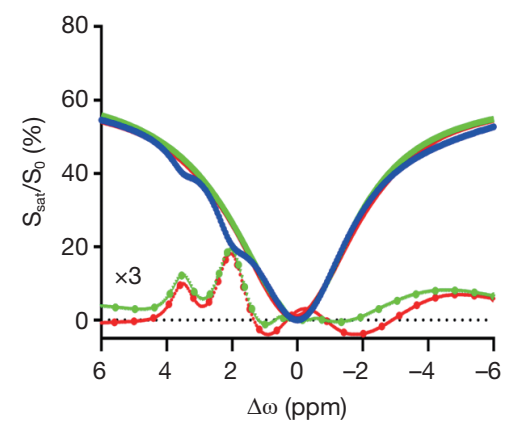

$1.5 \mu \mathrm{T}$

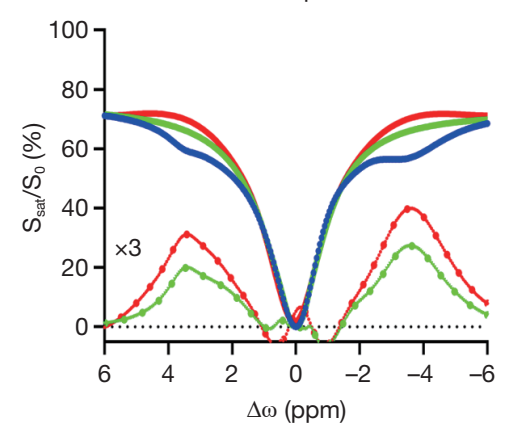

$3.0 \mu \mathrm{T}$

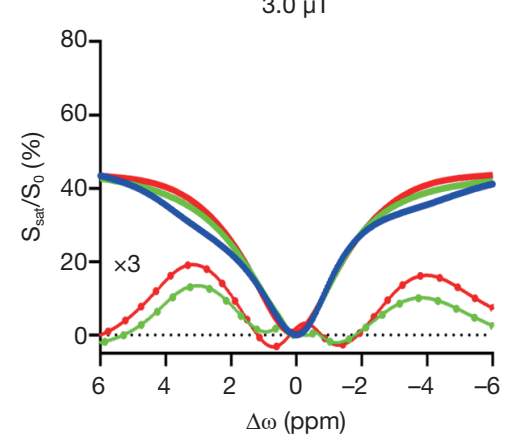

Figure 3 Comparison of VOPVP fitting and LD fitting using simulating Z-spectra, for $\mathrm{B}_{1 \text {-sat }}$ of $0.5,1,1.5,2,2.5$ and $3 \mu \mathrm{T}$, respectively. (A) The 5-pool Bloch-simulation; (B) the 6-pool Bloch-simulation. 
Table 2 Comparison of fitting quality of LD and VOPVP in simulation

\begin{tabular}{|c|c|c|c|c|c|c|c|c|}
\hline Number of pools & Quantification quality & $\mathrm{B}_{1 \_ \text {sat }}(\mu \mathrm{T})$ & 0.5 & 1 & 1.5 & 2 & 2.5 & 3 \\
\hline \multirow{3}{*}{5} & & VOPVP & 0.0365 & 0.1355 & 0.1202 & 0.0744 & 0.0498 & 0.0332 \\
\hline & R-square & LD & 0.9865 & 0.9479 & 0.9535 & 0.9744 & 0.9884 & 0.9951 \\
\hline & & VOPVP & 0.9955 & 0.9834 & 0.9853 & 0.9905 & 0.9951 & 0.9959 \\
\hline \multirow{3}{*}{6} & & VOPVP & 0.0155 & 0.0309 & 0.0305 & 0.0196 & 0.0157 & 0.012 \\
\hline & R-square & LD & 0.9195 & 0.6636 & 0.6665 & 0.7901 & 0.8871 & 0.9427 \\
\hline & & VOPVP & 0.9908 & 0.9817 & 0.9821 & 0.9884 & 0.9907 & 0.9928 \\
\hline
\end{tabular}

LD, Lorentzian difference; VOPVP, Voxel-wise Optimization of Pseudo Voigt Profile; SSE, sum of squares due to error.

(Figure S2, Table S2).

\section{Tumor mouse}

Further, VOPVP fitting was applied to quantify the contributions of APT, glutamate, amine and NOE effects in a mouse bearing glioblastoma. The $\mathrm{T}_{2} \mathrm{w}$ image (Figure $6 \mathrm{~A}$ ) indicated the anatomy, with a tumor ROI and the contralateral control one marked. To visualize the quantified spectra for all voxels, the offset-cut images were plotted with the residual spectra of LD fitting and VOPVP (Figure 6B,C). Transverse coordinates between 56 and 66 are the location of the tumor. Notably, the offset-cut values of VOPVP fitting were significantly higher at $3.5 \mathrm{ppm}$ and $2 \mathrm{ppm}$ in tumor than those in normal contralateral tissue. In contrast, the offset-cut values of LD fitting were significantly higher for Amide $3.5 \mathrm{ppm}$, Glu-CEST 3 ppm, Guanidinium-Amine $2 \mathrm{ppm}$ and lower for NOE between -2 and $-5 \mathrm{ppm}$ in the tumor than those in normal contralateral tissue. The differences between VOPVP fitting and LD fitting for tumor ROI and normal contralateral ROI at three $\mathrm{B}_{1 \_ \text {sat }}$ level $(0.8,1.2$ and $2.4 \mu \mathrm{T})$ are presented in Figure $6 \mathrm{D}, \mathrm{E}$. Moreover, the dips centered at $-3.5,2$ and 3.5 ppm could be clearly observed on the $\mathrm{Z}$-spectra at relatively low $\mathrm{B}_{1_{\text {_sat }} \text {, }}$ which corresponded to aliphatic NOE, guanidine amine and amide, respectively. Another dip around -1.6 ppm was observed in normal tissues, but not in tumors with low $\mathrm{B}_{1 \_ \text {sat }}$, which were consistent with previous reports $(58,59)$. Theoretically, a dip at $3 \mathrm{ppm}$ from Glu-CEST should be visible at high $B_{1 \_ \text {sat }}(10)$. However, it was not obviously shown on the Z-spectra due to the broadened dips. The residual of both VOPVP and LD fitting for the
ROIs of tumor and normal contralateral tissue appeared at $3.5 \mathrm{ppm}$ (amide), downfield from water and 2-5 ppm (NOE) up field from water. Overall, the fitting curves of VOPVP for the two ROIs were closer to the experimental measurements compared to those obtained by LD. Besides, the overestimation of LD fitting becomes obvious with the increasing $\mathrm{B}_{1 \_ \text {sat, }}$, which was also observed in a recent CEST study of simulations at $9.4 \mathrm{~T}$ (40). The peaks appeared at $2 \mathrm{ppm}$ could be clearly observed on the $\mathrm{MTR}_{\text {asym }}$ map (Figure $6 \mathrm{~F}$ ) at all $\mathrm{B}_{1_{-} \text {sat }}$, which were consistent with the conclusion drawn from the fitted residual spectra.

Furthermore, the proposed VOPVP method was evaluated by comparing with LD fitting and $M_{T R}$ asym (3.5 ppm) (Figure 7). Figure 7 shows the fitted amplitude maps using $\mathrm{MTR}_{\text {asym }}$ at $3.5 \mathrm{ppm}$, LD fitting and VOPVP fitting in a mouse tumor model. The images were fitted voxel-wise to Eq. [2] by using the nonlinear fitting function (lsqcurvefit) in MATLAB. In line with previous findings on the APT imaging of glioma (4,60-62), a remarkably higher APT effect was found in tumors fitted by VOPVP. For NOE (VOPVP fitting), our results demonstrated the intensity of tumor was increased at higher $\mathrm{B}_{1 \_ \text {sat }}(1.2$ and $2.4 \mu \mathrm{T})$ compared to normal tissue, but decreased at lower $\mathrm{B}_{1_{\_} \text {sat }}$ $(0.8 \mu \mathrm{T})$. Moreover, VOPVP fitting revealed a pronounced positive contrast in the tumor analyzed by Glu-CEST map. Whilst, the NOE (LD fitting) in tumor was lower than that in normal tissue at $\mathrm{B}_{1 \_ \text {sat }}=0.8 \mu \mathrm{T}$, which were consistent with previous findings (63). Additionally, $\mathrm{MTR}_{\text {asym }}$ $(3.5 \mathrm{ppm})$ indicated that the signal intensity of tumor location was significantly higher than that of normal contralateral tissue location, which was in accordance with the results of previous studies $(4,63,64)$. 
A
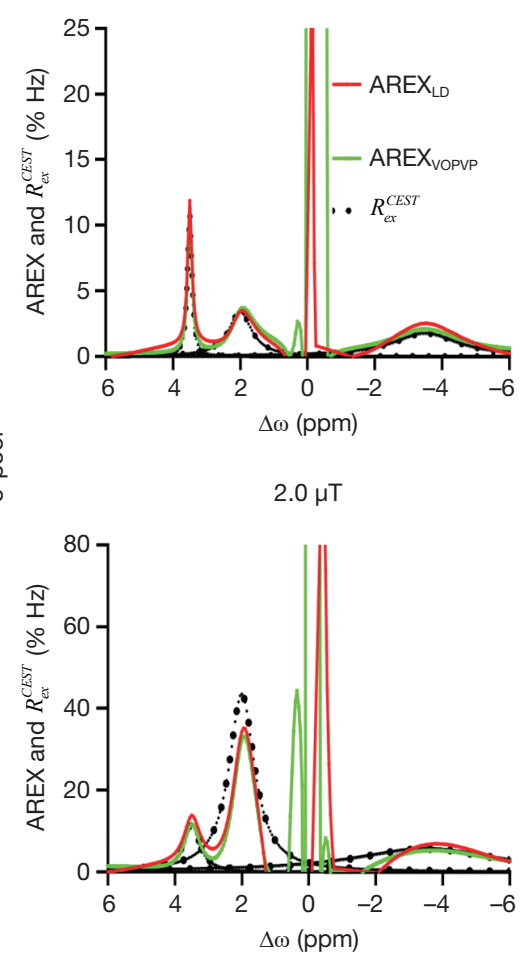

B
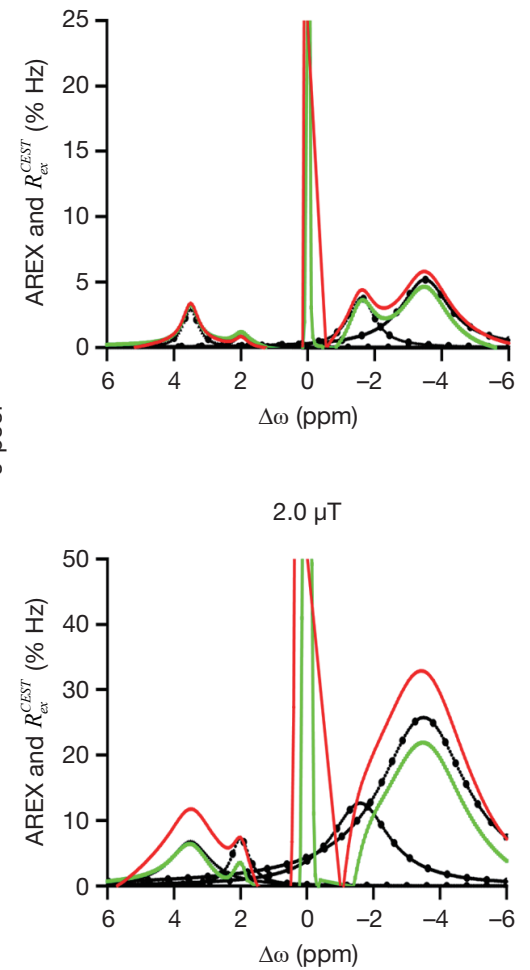

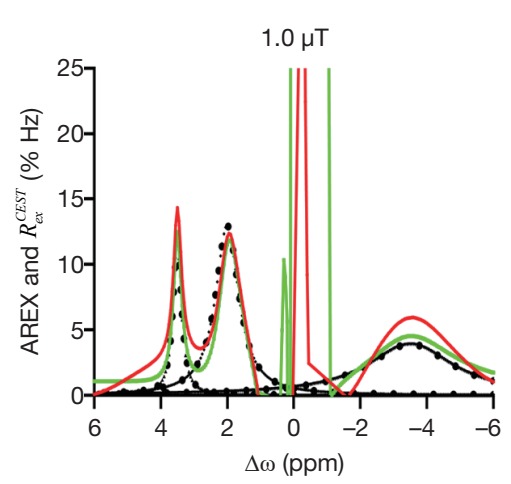

$2.5 \mu \mathrm{T}$

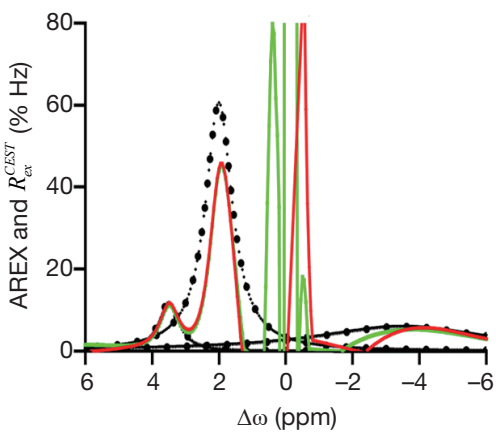

$1.0 \mu \mathrm{T}$

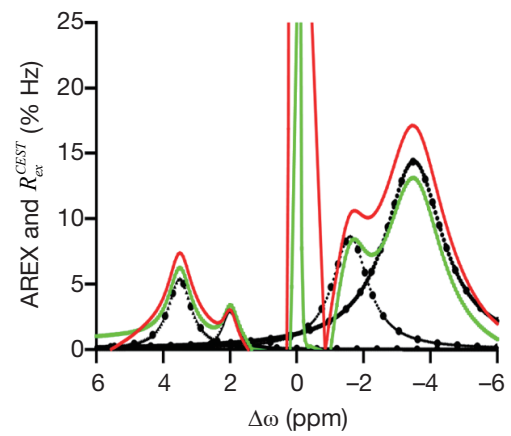

$2.5 \mu \mathrm{T}$

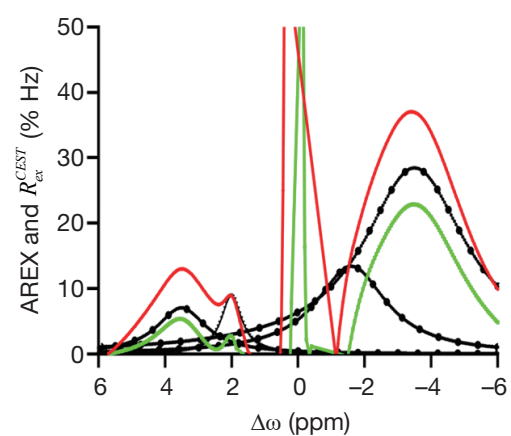

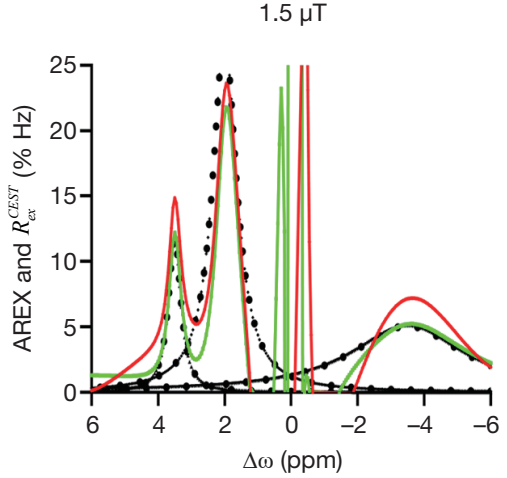

$3.0 \mu \mathrm{T}$
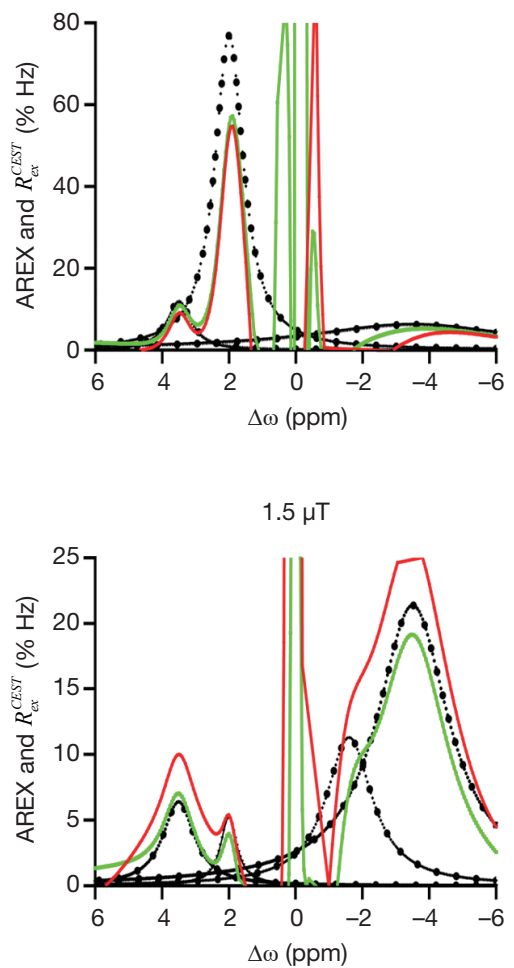

$3.0 \mu \mathrm{T}$

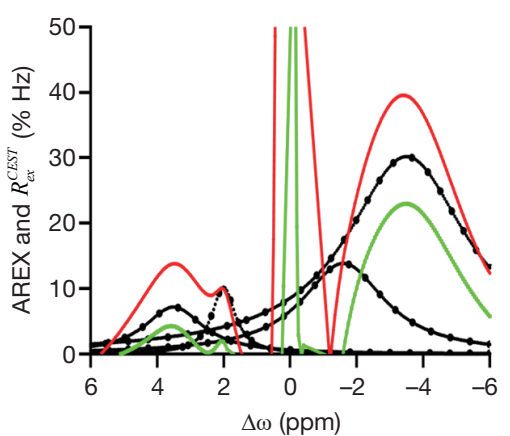

Figure 4 Quantification of the fitted Z-spectra using AREX (AREX $\mathrm{LD}_{\mathrm{LD}}$ and $\mathrm{AREX} \mathrm{X}_{\mathrm{VOPVP}}$ ), and comparison with the analytical $R_{e x}^{\text {CEST }}$ spectra, for $\mathrm{B}_{1 \text {-sat }}$ of $0.5,1,1.5,2,2.5$ and $3 \mu \mathrm{T}$, respectively. (A) The 5-pool Bloch-simulation; (B) the 6-pool Bloch-simulation. 
Table 3 Comparison of quantified peaks using LD and VOPVP with the analytical $R_{e x}^{C E S T}$ as a gold standard (40)

\begin{tabular}{|c|c|c|c|c|}
\hline Variable & Amide & $\begin{array}{l}\text { Intermediate exchanging } \\
\text { amine }\end{array}$ & NOE (-1.6 ppm) & NOE (-3.5 ppm) \\
\hline $\mathrm{AREX}_{\mathrm{LD}} / R_{e x}^{C E S T}$ (5-pool) & $1.1,1.2,1.3,1.2,1.0,0.8$ & $1.0,0.9,0.9,0.8,0.7,0.7$ & - & $1.4,1.5,1.4,1.2,0.8,0.4$ \\
\hline $\mathrm{AREX}_{\mathrm{VOPVP}} / R_{e x}^{C E S T}(5-\mathrm{pool})$ & $1.0,1.0,1.0,1.0,0.9,0.9$ & $1.0,0.9,0.8,0.7,0.7,0.7$ & - & $1.1,1.1,1.0,1.0,1.0,1.0$ \\
\hline $\operatorname{AREX} \mathrm{LD}_{\mathrm{D}} / R_{e x}^{C E S T}$ (6-pool) & $1.8,2.3,2.7,2.9,3.0,3.0$ & $2.8,2.6,2.4,2.2,2.1,2.0$ & $1.4,1.5,1.6,1.7,1.8,1.8$ & $1.5,1.5,1.5,1.6,1.6,1.6$ \\
\hline
\end{tabular}

$B_{1 \text {-sat }}=0.5,1,1.5,2,2.5,3 \mu \mathrm{T}$. AREX, apparent exchanged-dependent relaxation; LD, Lorentzian difference; VOPVP, Voxel-wise Optimization of Pseudo Voigt Profile.
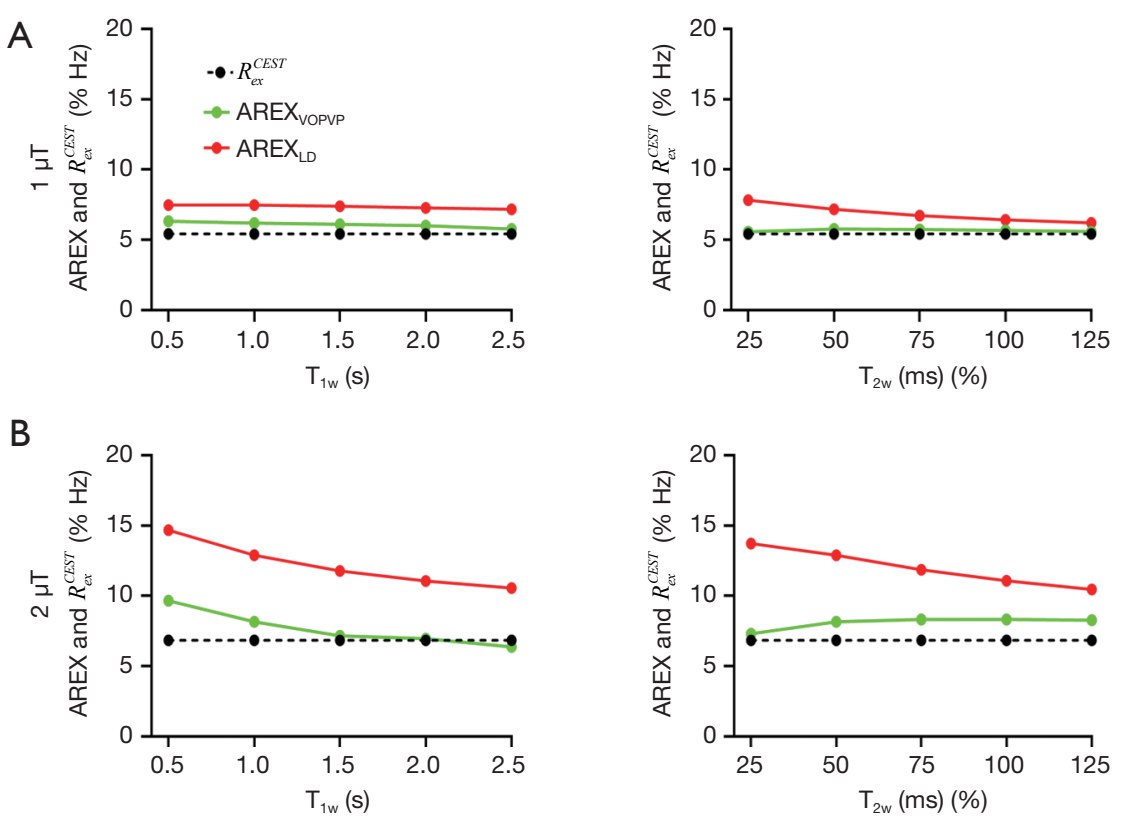

Figure $5 \mathrm{AREX}_{\mathrm{LD}}, \mathrm{AREX}_{\mathrm{VOPVP}}$ and calculated $R_{e x}^{C E S T}$ for APT from simulated $\mathrm{Z}$-spectra with variation of $\mathrm{T}_{1} \mathrm{w}$ and $\mathrm{T}_{2} \mathrm{w}$, with the $\mathrm{B}_{1 \_ \text {sat }}$ of $(\mathrm{A})$ $1 \mu \mathrm{T}$, (B) $2 \mu \mathrm{T}$.

Table 4 describes the quality of each CEST map. For APT and Glu-CEST, VOPVP method exhibited higher CNR than LD method and $\mathrm{MTR}_{\text {asym }}(3.5 \mathrm{ppm})$, when $\mathrm{B}_{1 \text { _sat }}$ $>1 \mu \mathrm{T}$.

\section{Discussion}

In this study, we propose a novel CEST quantification method, namely VOPVP, by fitting $\mathrm{Z}$-spectra with a linear combination of Gaussian- and Lorentzian-line shapes as the reference spectra and taking the difference among experimental data for CEST quantification. To improve the conventional LD and enhance its robustness for various occasions (e.g., larger $\mathrm{B}_{1_{\text {_sat }}}$ and greater MT), we not only modified the fitting function from Lorentzian to Pseudo Voigt Profile (PVP), but also integrated a $B_{1}$-dependent optimization for better compensation of the altered DS (spillover effect) and MT under different $\mathrm{B}_{1 \_ \text {sat }}$. The main purposes of $Z$-spectra fitting in Lorentzian and VOPVP are to generate a reference spectrum without CEST and NOE, and to best simulate the confounding MT and DS. The semisolid MT effect is asymmetric around the water peak, which induces signal decline over a wide frequency range. It has been reported that a baseline term against the Lorentzian analysis can minimize the impact of global MT effect and noise arising from motion (40,50). A previous 

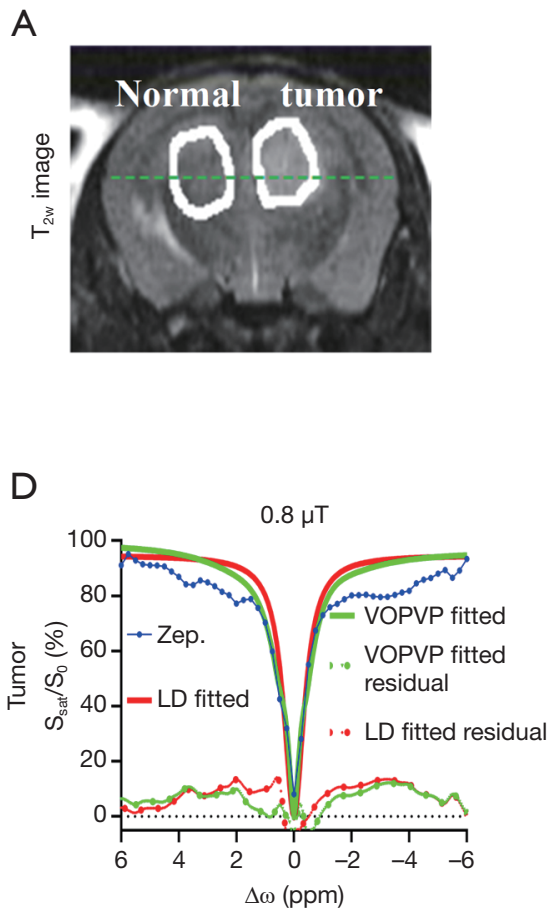

E

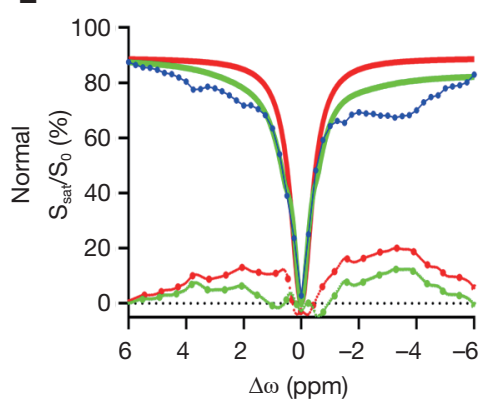

F

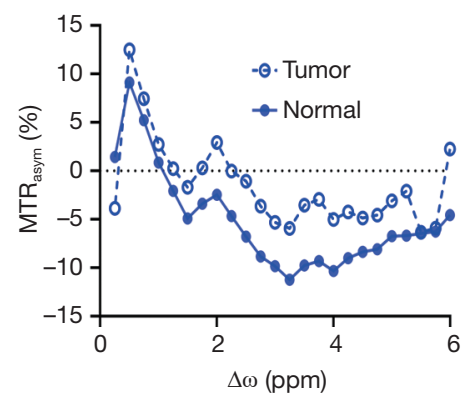

B
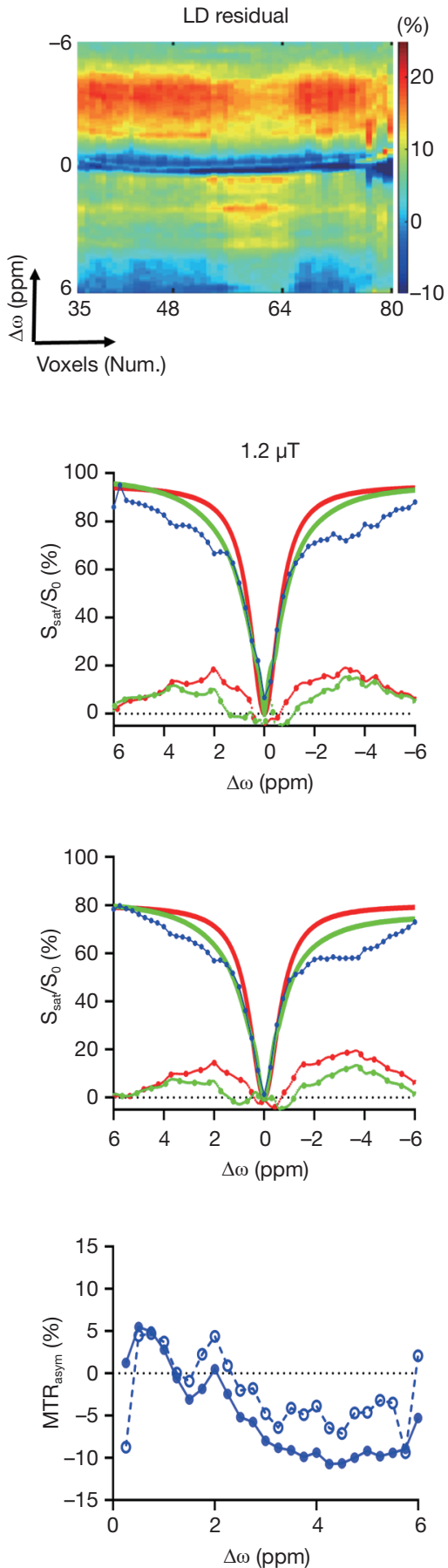

C
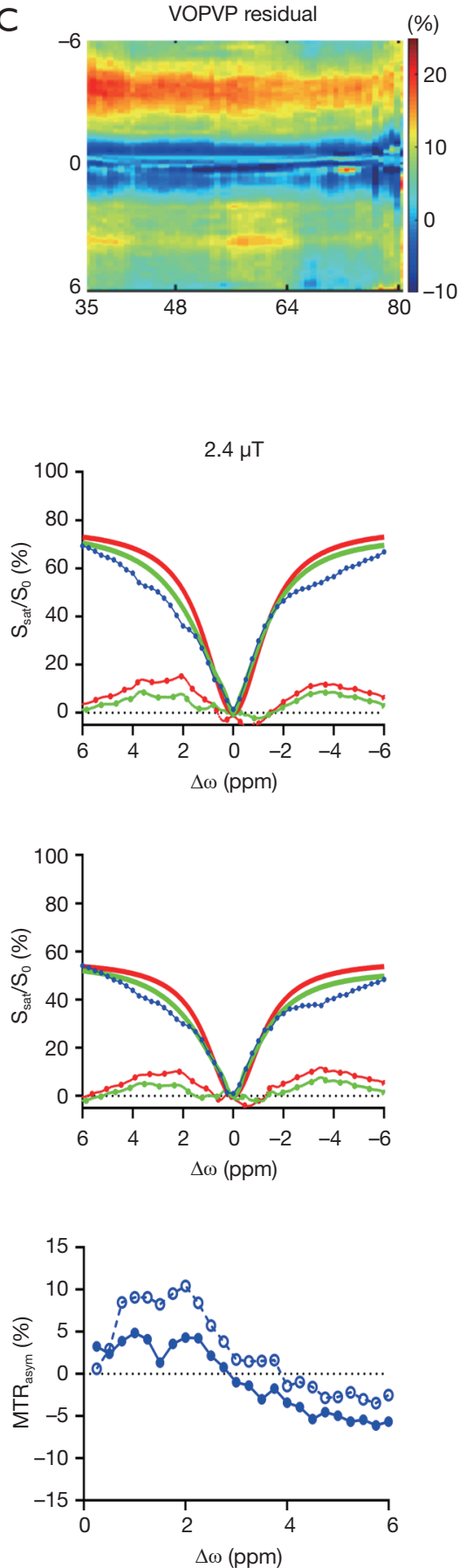

Figure 6 Quantitative assessment of in vivo tumor-bearing mouse brain. (A) The $\mathrm{T}_{2} \mathrm{w}$ image, marked with a tumor ROI and the contralateral control for spectral analysis. (B) The offset-cut map using residual spectra of LD fitting for B1_sat $=1.2 \mu \mathrm{T}$, which locations are indicated by dash line in (A) of $\mathrm{T}_{2} \mathrm{w}$ image. (C) The offset-cut map using residual spectra of VOPVP for B1_sat $=1.2 \mu \mathrm{T}$, which locations are indicated by dash line in (A) of $\mathrm{T}_{2} \mathrm{w}$ image. (D) Comparison of VOPVP fitting and LD fitting for tumor region. (E) Comparison of VOPVP fitting and LD fitting for the normal contralateral region. (F) MTR $_{\text {asym }}$ analysis both for the tumor region and the normal contralateral region. VOPVP, Voxel-wise Optimization of Pseudo Voigt Profile; LD, Lorentzian difference; MTR $_{\text {asym }}$, magnetization transfer ratio asymmetry. 


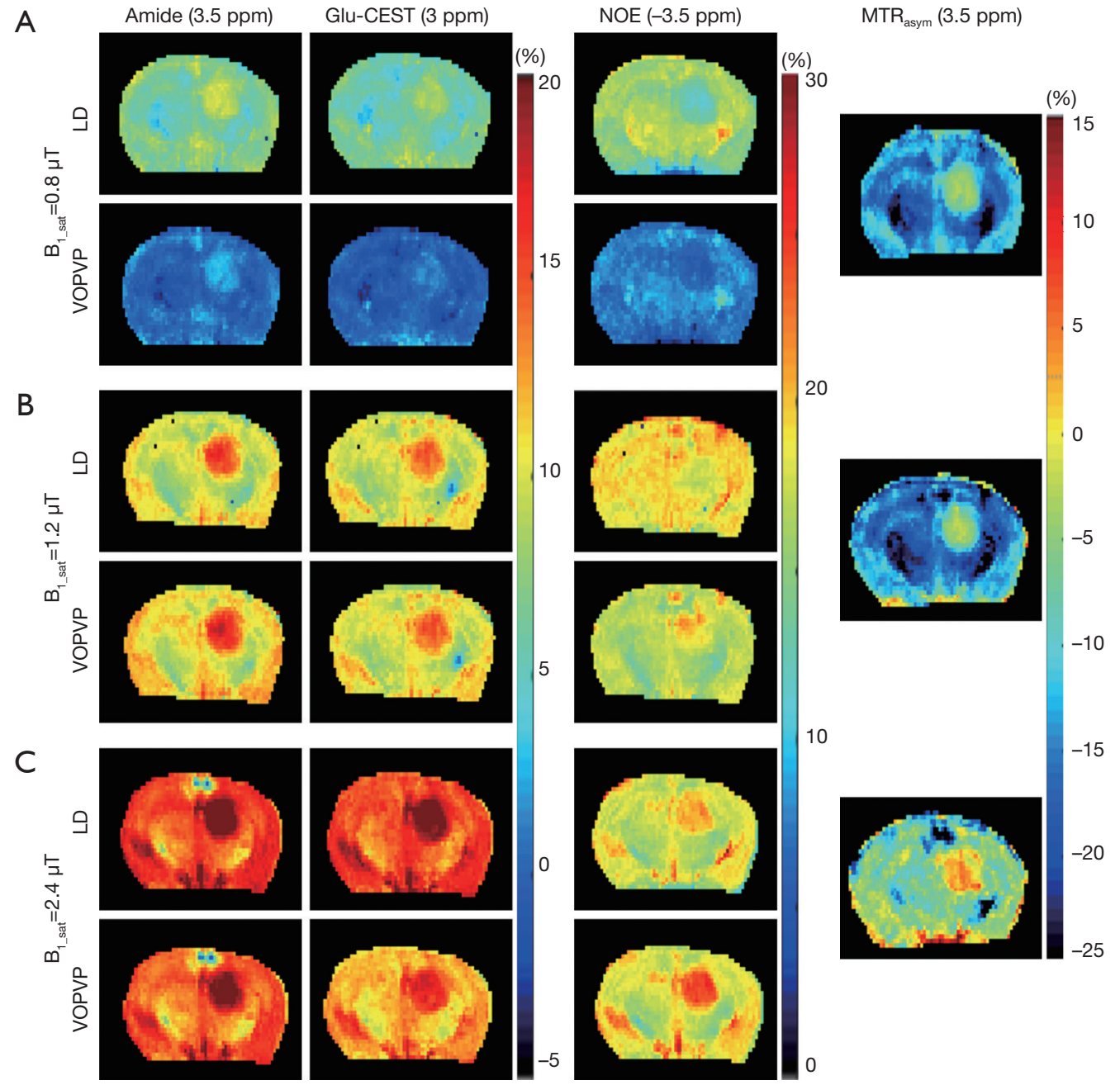

Figure 7 Comparison of fitted amplitudes maps using VOPVP fitting, Voxel-wise LD fitting and MTR $_{\text {asym }}(3.5$ ppm) in a representative mouse brain, for $\mathrm{B}_{1 \_ \text {sat }}$ of (A) $0.8 \mu \mathrm{T}$, (B) $1.2 \mu \mathrm{T}$ and (C) $2.4 \mu \mathrm{T}$. VOPVP, Voxel-wise Optimization of Pseudo Voigt Profile; LD, Lorentzian difference; $\mathrm{MTR}_{\text {asym }}$, magnetization transfer ratio asymmetry.

study has renormalized CEST Z-spectra by averaging the signal intensity of both end of $\mathrm{Z}$-spectra of at relatively lower $B_{1 \_ \text {sat }}(18)$ before performing LD fitting. Similarly, in our VOPVP method, a compensate ratio factor was applied to the experimental $Z$-spectra data, according to the acquisition $\mathrm{B}_{1 \text { ssat. }}$. The relationship between the ratio and $\mathrm{B}_{1 \text { sat }}$ was established in advance through an offline step using the 5-pool Bloch simulation (Figures 1,2). Both simulation and in vivo mouse revealed that VOPVP displayed a better approximation to the referenced part of $\mathrm{Z}$-spectra in comparison with LD analysis. Despite that VOPVP outperformed the conventional LD when the acquisition $B_{1 \text { _sat }}$ is larger for fast-exchangeable species or when the tissue imposes a greater MT contribution, this method does not work well with larger $B_{1 \_ \text {sat }}$ and greater MT component, especially not appropriate for amines and NOE (-1.6 ppm) that are closer to water (Table 3).

In the proposed approach we applied a B1-dependent adjustment to the measured $\mathrm{Z}$-spectra, before starting of the optimization of pseudo voigt profile. The equation for adjustment was obtained offline, through simulations using a previously-published 5-pool model for brain tissue at 9.4T. Accurate reference spectra describing DS and MT were determined using a 2-pool model (water and semisolid), while Lorentzian fitting was also performed. The inaccuracy of LD fitting was further defined by the ratio of two residual areas, corresponding to the Lorentzian fitting spectrum and the accurate reference spectrum. As seen in Figure 2, 
Table 4 Comparison of CNR between MTR $_{\text {asym }}(3.5 \mathrm{ppm})$, LD fitting and VOPVP fitting in tumor mouse brain at 11.7T

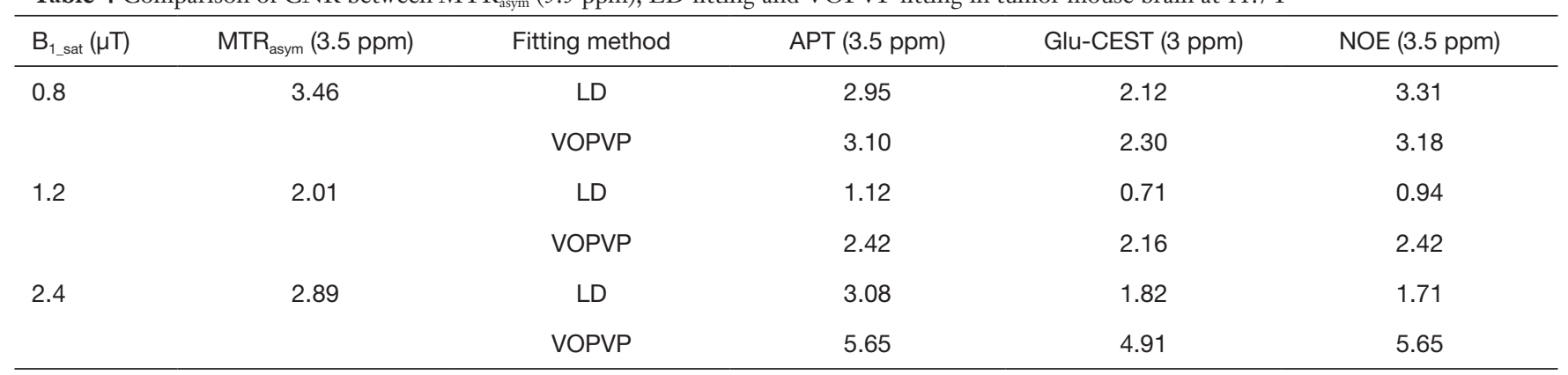

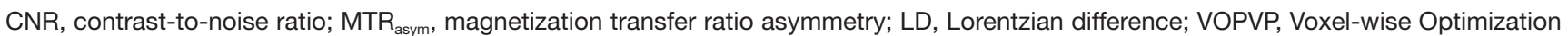
of Pseudo Voigt Profile; APT, amide proton transfer; CEST, chemical exchange saturation transfer; NOE, nuclear overhauser enhancement.

when $B_{1 \text {-sat }}<1 \mu \mathrm{T}$, the ratio is close to 1 , indicating accurate quantification of LD. But, for larger $\mathrm{B}_{1 \text {-sat }}$, LD inaccurately quantified CEST signal with ratio $>1$. We also figured the linear relationships between ratios and $B_{1-\text { sat }}$. This prior knowledge of inaccuracy of $\mathrm{LD}$ at different $\mathrm{B}_{1_{-} \text {sat, }}$ was then employed in the online optimization step, to produce an adjusted $Z$-spectra better representing the DS and MT contributions under applied $\mathrm{B}_{1_{\text {_sat }}}$. In another word, our quantification approach employed the prior knowledge of $\mathrm{LD}$ mismatch at different $\mathrm{B}_{1 \_ \text {sat }}$ to constrain the optimization algorithm (Levenberg-Marquardt algorithm here), leading to more accurate quantification. Despite that the adjustment uses Ratios that calculated from a 5-pool model of brain tissue, the followed VOPVP optimization will make it work for a range of tissue parameters $\left(\mathrm{T}_{1} \mathrm{w}\right.$ and $\left.\mathrm{T}_{2} \mathrm{w}\right)$, as shown in Figures 3,4,5. The Ratios is also applicable for different filed strengths, as validated by the $3 \mathrm{~T}$ simulations (Figures S1,S2, Table S1). This constrained optimization method, through learning from the off-line simulations, could also applied to other fitting methods for CEST MRI in future.

Apart from taking the difference between a fitting reference spectra and the experimental data, several other quantification methods have been proposed, including apparent exchange-dependent relaxation (AREX) (5), extrapolated semi-solid magnetization transfer reference (EMR) (4,65), multi-pool Bloch-McConnell (BM) fitting (32), three-offset approach $(23,66)$ and the combination of these quantitative methods $(20,23,35,51)$. Superior to $\mathrm{MTR}_{\text {asym }}$, all these methods allow the separation of CEST and NOE effect. However, the accuracy and robustness of these methods are varied, and each has its own limitations. For instance, AREX has been applied only to slow- (e.g., APT) $(27,38)$ and intermediate-exchangeable [e.g., creatine (creCEST) (67)] solutes, which is also a fitting approach to quantify fast-exchangeable amine CEST signals $(5,27)$. BM fitting requires prior knowledge of parameters (e.g., $\mathrm{T}_{2}$ and $\mathrm{k}_{\mathrm{ex}}$ of each exchanging proton) and is time consuming compared to LD fitting and AREX (68). EMR approach has the potential to be an important and accurate CEST MRI quantitative technique (4), but it may overestimate the measurements of APT and NOE (4). Three-offset method is a relatively simple quantification approach $(23,66)$. However, the linear assumption underlying the three-offset method may be oversimplified $(39,40)$.

NOEs are depended on the applied $B_{1_{\text {_s sat }}}$ and filed strength. It has been reported there is no significant difference in the NOE $(9.4 \mathrm{~T}, 1 \mu \mathrm{T})$ between tumors and normal contralateral tissues (23). Previous findings have shown that the NOE signal at 4.7 Tesla in tumor is lower than that in normal contralateral tissue (63), which are consistent with the results of offset-cuts (Figure 6B,C). APT imaging, a specific form of CEST, can potentially serve as a non-invasive means to characterize abnormal tissues such as tumors (23). Previous reports have shown that the APTrelated pool size of the tumor center and rim is significantly larger than that of normal tissue, as opposed to the NOErelated pool size. Moreover, APT can detect malignant tumors $(63,64,69)$, the changes that have been induced by elevated mobile cellular and peptide contents (70). In the present study, it can be seen that the intensity of APT signal is higher in tumor than in normal contralateral tissue, as revealed the offset-cuts (Figure 6B,C) and fitted amplitudes maps (Figure 7) of our proposed VOPVP fitting, which is consistent with the findings of previous literature $(4,70)$.

As an improvement of LD method, our VOPVP fitting may provide a simple, robust and more accurate approach for quantifying CEST and NOE contrast. More in vivo validations and at the clinical field strength will be performed 
in the future.

\section{Acknowledgments}

The authors would like to thank Dr. Chengyan Chu and Dr. Piotr Walczak at Johns Hopkins University for their generous offering of a tumor-bearing mouse.

Funding: This work was supported by the National Natural Science Foundation of China under Grant No.61601364, Xi'an Key Laboratory of Radiomics and Intelligent Perception Grant 201805060ZD11CG44, and by the Postgraduate Independent Innovation Project of Northwest University (China) Grant YZZ17180. In vivo MRI data of the mouse were collected under support of Johns Hopkins Radiology Britestar Award to Dr. Xiaolei Song.

\section{Footnote}

Conflicts of Interest: The authors have no conflicts of interest to declare.

Ethical Statement: MR imaging experiment on a tumorbearing mouse was performed under the approval of the Johns Hopkins University Animal Care and Use Committee (ACUC) with protocol \# MO13M251.

Disclaimer: The content is solely the responsibility of the author and does not necessarily represent the official views of the funding agencies.

\section{References}

1. Ward KM, Aletras AH, Balaban RS. A new class of contrast agents for MRI based on proton chemical exchange dependent saturation transfer (CEST). J Magn Reson 2000;143:79-87.

2. Zhou J, vanZijl PC. Chemical exchange saturation transfer imaging and spectroscopy. Prog Nucl Magn Reson Spectrosc 2006;48:109-36.

3. van Zijl PC, Yadav NN. Chemical exchange saturation transfer (CEST): what is in a name and what isn't? Magn Reson Med 2011;65:927-48.

4. Heo HY, Zhang Y, Lee DH, Hong X, Zhou, J. Quantitative assessment of amide proton transfer (APT) and nuclear overhauser enhancement (NOE) imaging with extrapolated semi-solid magnetization transfer reference (EMR) signals: Application to a rat glioma model at 4.7 Tesla. Magn Reson Med 2016;75:137-49.
5. Zhang XY, Wang F, Li H, Xu J, Gochberg DF, Gore JC, $\mathrm{Zu} \mathrm{Z}$. CEST imaging of fast exchanging amine pools with corrections for competing effects at 9.4 T. NMR Biomed 2017;30. doi: 10.1002/nbm.3715.

6. Zhou J, Lal B, Wilson DA, Laterra J, van Zijl PC. Amide proton transfer (APT) contrast for imaging of brain tumors. Magn Reson Med 2003;50:1120-6.

7. Cai K, Singh A, Poptani H, Li W, Yang S, Lu Y, Hariharan H, Zhou XJ, Reddy R. CEST signal at 2ppm (CEST@2ppm) from Z-spectral fitting correlates with creatine distribution in brain tumor. NMR Biomed 2015;28:1-8.

8. Chan KW, McMahon MT, Kato Y, Liu G, Bulte JW, Bhujwalla ZM, Artemov D, van Zijl PC. Natural D-glucose as a biodegradable MRI contrast agent for detecting cancer. Magn Reson Med 2012;68:1764-73.

9. Walker-Samuel S, Ramasawmy R, Torrealdea F, Rega M, Rajkumar V, Johnson SP, Richardson S, Goncalves M, Parkes HG, Arstad E, Thomas DL, Pedley RB, Lythgoe MF, Golay X. In vivo imaging of glucose uptake and metabolism in tumors. Nat Med 2013;19:1067-72.

10. Cai K, Haris M, Singh A, Kogan F, Greenberg JH, Hariharan H, Detre JA, Reddy R. Magnetic resonance imaging of glutamate. Nat Med 2012;18:302-6.

11. van Zijl PC, Jones CK, Ren J, Malloy CR, Sherry AD. MRI detection of glycogen in vivo by using chemical exchange saturation transfer imaging (glycoCEST). Proc Natl Acad Sci U S A 2007;104:4359-64.

12. Haneder S, Apprich SR, Schmitt B, Michaely HJ, Schoenberg SO, Friedrich KM, Trattnig S. Assessment of glycosaminoglycan content in intervertebral discs using chemical exchange saturation transfer at 3.0 Tesla: preliminary results in patients with low-back pain. Eur Radiol 2013;23:861-8.

13. Zhang S, Malloy CR, Sherry AD. MRI Thermometry Based on PARACEST Agents. J Am Chem Soc 2005;127:17572-3.

14. Guo Y, Zhou IY, Chan ST, Wang Y, Mandeville ET, Igarashi T, Lo EH, Ji X, Sun PZ. pH-sensitive MRI demarcates graded tissue acidification during acute stroke - pH specificity enhancement with magnetization transfer and relaxation-normalized amide proton transfer (APT) MRI. Neuroimage 2016;141:242-9.

15. Sun PZ, Wang E, Cheung JS. Imaging acute ischemic tissue acidosis with $\mathrm{pH}$-sensitive endogenous amide proton transfer (APT) MRI--correction of tissue relaxation and concomitant RF irradiation effects toward mapping quantitative cerebral tissue pH. Neuroimage 2012;60:1-6. 
16. Longo DL, Cutrin JC, Michelotti F, Irrera P, Aime S. Noninvasive evaluation of renal $\mathrm{pH}$ homeostasis after ischemia reperfusion injury by CEST-MRI. NMR Biomed 2017;30. doi: 10.1002/nbm.3720.

17. Jeong HK, Han K, Zhou J, Zhao Y, Choi YS, Lee SK, Ahn SS. Characterizing amide proton transfer imaging in haemorrhage brain lesions using 3T MRI. Eur Radiol 2017;27:1577-84.

18. Tietze A, Blicher J, Mikkelsen IK, Østergaard L, Strother MK, Smith SA, Donahue MJ. Assessment of ischemic penumbra in patients with hyperacute stroke using amide proton transfer (APT) chemical exchange saturation transfer (CEST) MRI. NMR Biomed 2014;27:163-74.

19. Sun PZ, Cheung JS, Wang E, Lo EH. Association between $\mathrm{pH}$-weighted endogenous amide proton chemical exchange saturation transfer MRI and tissue lactic acidosis during acute ischemic stroke. J Cereb Blood Flow Metab 2011;31:1743-50.

20. Zaiss M, Xu J, Goerke S, Khan IS, Singer RJ, Gore JC, Gochberg DF, Bachert P. Inverse Z-spectrum analysis for spillover-, MT-, and T1 -corrected steady-state pulsed CEST-MRI--application to $\mathrm{pH}$-weighted MRI of acute stroke. NMR Biomed 2014;27:240-52.

21. Dula AN, Arlinghaus LR, Dortch RD, Dewey BE, Whisenant JG, Ayers GD, Yankeelov TE, Smith SA. Amide proton transfer imaging of the breast at $3 \mathrm{~T}$ : establishing reproducibility and possible feasibility assessing chemotherapy response. Magn Reson Med 2013;70:216-24.

22. Jia G, Abaza R, Williams JD, Zynger DL, Zhou J, Shah ZK, Patel M, Sammet S, Wei L, Bahnson RR, Knopp MV. Amide proton transfer MR imaging of prostate cancer: a preliminary study. J Magn Reson Imaging 2011;33:647-54.

23. Xu J, Zaiss M, Zu Z, Li H, Xie J, Gochberg DF, Bachert P, Gore JC. On the origins of chemical exchange saturation transfer (CEST) contrast in tumors at 9.4 T. NMR Biomed 2014;27:406-16.

24. Desmond KL, Moosvi F, Stanisz GJ. Mapping of amide, amine, and aliphatic peaks in the CEST spectra of murine xenografts at 7 T. Magn Reson Med 2014;71:1841-53.

25. Li C, Peng S, Wang R, Chen H, Su W, Zhao X, Zhou J, Chen M. Chemical exchange saturation transfer MR imaging of Parkinson's disease at 3 Tesla. Eur Radiol 2014;24:2631-9.

26. Sagiyama K, Mashimo T, Togao O, Vemireddy V, Hatanpaa KJ, Maher EA, Mickey BE, Pan E, Sherry AD, Bachoo RM, Takahashi M. In vivo chemical exchange saturation transfer imaging allows early detection of a therapeutic response in glioblastoma. Proc Natl Acad Sci U S A 2014;111:4542-7.

27. Zaiss M, Windschuh J, Paech D, Meissner JE, Burth S, Schmitt B, Kickingereder P, Wiestler B, Wick W, Bendszus M, Schlemmer HP, Ladd ME, Bachert P, Radbruch A. Relaxation-compensated CEST-MRI of the human brain at 7T: Unbiased insight into NOE and amide signal changes in human glioblastoma. Neuroimage 2015;112:180-8.

28. Jiang S, Zou T, Eberhart CG, Villalobos MAV, Heo HY, Zhang Y, Wang Y, Wang X, Yu H, Du Y, van Zijl PCM, Wen Z, Zhou J. Predicting IDH mutation status in grade II gliomas using amide proton transfer-weighted (APTw) MRI. Magn Reson Med 2017;78:1100-9.

29. Togao O, Yoshiura T, Keupp J, Hiwatashi A, Yamashita K, Kikuchi K, Suzuki Y, Suzuki SO, Iwaki T, Hata N, Mizoguchi M, Yoshimoto K, Sagiyama K, Takahashi M, Honda H. Amide proton transfer imaging of adult diffuse gliomas: correlation with histopathological grades. Neuro Oncol 2014;16:441-8.

30. Aime S, Barge A, Delli Castelli D, Fedeli F, Mortillaro A, Nielsen FU, Terreno E. Paramagnetic lanthanide(III) complexes as $\mathrm{pH}$-sensitive chemical exchange saturation transfer (CEST) contrast agents for MRI applications. Magn Reson Med 2002;47:639-48.

31. Togao O, Hiwatashi A, Yamashita K, Kikuchi K, Keupp J, Yoshimoto K, Kuga D, Yoneyama M, Suzuki SO, Iwaki T, Takahashi M, Iihara K, Honda H. Grading diffuse gliomas without intense contrast enhancement by amide proton transfer MR imaging: comparisons with diffusion- and perfusion-weighted imaging. Eur Radiol 2017;27:578-88.

32. Woessner DE, Zhang S, Merritt ME, Sherry AD. Numerical solution of the Bloch equations provides insights into the optimum design of PARACEST agents for MRI. Magn Reson Med 2005;53:790-9.

33. Song X, Gilad AA, Joel S, Liu G, Bar-Shir A, Liang Y, Gorelik M, Pekar JJ, van Zijl PC, Bulte JW, McMahon MT. CEST phase mapping using a length and offset varied saturation (LOVARS) scheme. Magn Reson Med 2012;68:1074-86.

34. Zhou J, Wilson DA, Sun PZ, Klaus JA, Van Zijl PC. Quantitative description of proton exchange processes between water and endogenous and exogenous agents for WEX, CEST, and APT experiments. Magn Reson Med 2004;51:945-952.

35. Zu Z, Li H, Xu J, Zhang XY, Zaiss M, Li K, Does MD, Gore JC, Gochberg DF. Measurement of APT using a combined CERT-AREX approach with varying duty 
cycles. Magn Reson Imaging 2017;42:22-31.

36. Yuan J, Zhang Q, Wang YX, Wei J, Zhou J. Accuracy and uncertainty of asymmetric magnetization transfer ratio quantification for amide proton transfer (APT) imaging at 3T: a Monte Carlo study. Conf Proc IEEE Eng Med Biol Soc 2013;2013:5139-42.

37. Ji Y, Zhou IY, Qiu B, Sun PZ. Progress toward quantitative in vivo chemical exchange saturation transfer (CEST) MRI. Isr J Chem 2017;57:809-24.

38. Windschuh J, Zaiss M, Meissner JE, Paech D, Radbruch A, Ladd ME, Bachert P. Correction of B1-inhomogeneities for relaxation-compensated CEST imaging at $7 \mathrm{~T}$. NMR Biomed 2015;28:529-37.

39. Zhou IY, Wang E, Cheung JS, Zhang X, Fulci G, Sun PZ. Quantitative chemical exchange saturation transfer (CEST) MRI of glioma using Image Downsampling Expedited Adaptive Least-squares (IDEAL) fitting. Sci Rep 2017;7:84.

40. Zhang XY, Wang F, Li H, Xu J, Gochberg DF, Gore JC, $\mathrm{Zu} \mathrm{Z}$. Accuracy in the quantification of chemical exchange saturation transfer (CEST) and relayed nuclear Overhauser enhancement (rNOE) saturation transfer effects. NMR Biomed 2017;30. doi: 10.1002/nbm.3716.

41. Zaiss M, Schmitt B, Bachert P. Quantitative separation of CEST effect from magnetization transfer and spillover effects by Lorentzian-line-fit analysis of z-spectra. J Magn Reson 2011;211:149-55.

42. Liu G, Gilad AA, Bulte JW, van Zijl PC, McMahon MT. High-throughput screening of chemical exchange saturation transfer MR contrast agents. Contrast Media Mol Imaging 2010;5:162-70.

43. Zaiss M, Bachert P. Chemical exchange saturation transfer (CEST) and MR Z-spectroscopy in vivo: a review of theoretical approaches and methods. Phys Med Biol 2013;58:R221-69.

44. Wertheim GK, Butler MA, West KW, Buchanan DNE. Determination of the Gaussian and Lorentzian content of experimental line shapes. Rev Sci Instrum 1974;45:1369-71.

45. Thompson P, Cox DE, Hastings JB. Rietveld refinement of Debye-Scherrer synchrotron X-ray data from Al2O3.J Appl Cryst 1987;20:79-83.

46. Di Roccoa HO, Cruzado A. The voigt profile as a sum of a gaussian and a lorentzian functions, when the weight coefficient depends only on the widths ratio. Acta Physica Polonica A 2012;122:666-9.

47. Ida T, Ando M, Toraya H. Extended pseudo-Voigt function for approximating the Voigt profile. J Appl Cryst 2000;33:1311-66.
48. Heo HY, Lee DH, Zhang Y, Zhao X, Jiang S, Chen M, Zhou J. Insight into the quantitative metrics of chemical exchange saturation transfer (CEST) imaging. Magn Reson Med 2017;77:1853-65.

49. Liu G, Moake M, Har-el YE, Long CM, Chan KW, Cardona A, Jamil M, Walczak P, Gilad AA, Sgouros G, van Zijl PC, Bulte JW, McMahon MT. In vivo multicolor molecular MR imaging using diamagnetic chemical exchange saturation transfer liposomes. Magn Reson Med 2012;67:1106-13.

50. Jones CK, Polders D, Hua J, Zhu H, Hoogduin HJ, Zhou J, Luijten P, van Zijl PC. In vivo three-dimensional wholebrain pulsed steady-state chemical exchange saturation transfer at 7 T. Magn Reson Med 2012;67:1579-89.

51. Li H, Li K, Zhang XY, Jiang X, Zu Z, Zaiss M, Gochberg DF, Gore JC, Xu J. R1 correction in amide proton transfer imaging: indication of the influence of transcytolemmal water exchange on CEST measurements. NMR Biomed 2015;28:1655-62.

52. Sun P, Xia S, Lal B, Eberhart CG, Quinones-Hinojosa A, Maciaczyk J, Matsui W, Dimeco F, Piccirillo SM, Vescovi AL, Laterra J. DNER, an epigenetically modulated gene, regulates glioblastoma-derived neurosphere cell differentiation and tumor propagation. Stem cells (Dayton, Ohio) 2009;27:1473-86.

53. Ying M, Wang S, Sang Y, Sun P, Lal B, Goodwin CR, Guerrero-Cazares H, Quinones-Hinojosa A, Laterra J, Xia S. Regulation of glioblastoma stem cells by retinoic acid: role for Notch pathway inhibition. Oncogene 2011;30:3454-67.

54. Kim M, Gillen J, Landman BA, Zhou J, van Zijl PC. Water saturation shift referencing (WASSR) for chemical exchange saturation transfer (CEST) experiments. Magn Reson Med 2009;61:1441-50.

55. Zaiss M, Zu Z, Xu J, Schuenke P, Gochberg DF, Gore JC, Ladd ME, Bachert P. A combined analytical solution for chemical exchange saturation transfer and semi-solid magnetization transfer. NMR Biomed 2015;28:217-230.

56. Li H, Zu Z, Zaiss M, Khan IS, Singer RJ, Gochberg DF, Bachert P, Gore JC, Xu J. Imaging of amide proton transfer and nuclear Overhauser enhancement in ischemic stroke with corrections for competing effects. NMR Biomed 2015;28:200-9.

57. Zaiss M, Bachert P. Exchange-dependent relaxation in the rotating frame for slow and intermediate exchange -modeling off-resonant spin-lock and chemical exchange saturation transfer. NMR Biomed 2013;26:507-18.

58. Zhang XY, Wang F, Jin T, Xu J, Xie J, Gochberg DF, 
Gore JC, Zu Z. MR imaging of a novel NOE-mediated magnetization transfer with water in rat brain at $9.4 \mathrm{~T}$. Magn Reson Med 2017;78:588-97.

59. Zhang XY, Wang F, Afzal A, Xu J, Gore JC, Gochberg DF, Zu Z. A new NOE-mediated MT signal at around -1.6ppm for detecting ischemic stroke in rat brain. Magn Reson Imaging 2016;34:1100-6.

60. Yuwen Zhou I, Wang E, Cheung JS, Lu D, Ji Y, Zhang X, Fulci G, Sun PZ. Direct saturation-corrected chemical exchange saturation transfer MRI of glioma: Simplified decoupling of amide proton transfer and nuclear overhauser effect contrasts. Magn Reson Med 2017;78:2307-14.

61. Salhotra A, Lal B, Laterra J, Sun PZ, van Zijl PC, Zhou J. Amide proton transfer imaging of 9L gliosarcoma and human glioblastoma xenografts. NMR Biomed 2008;21:489-97.

62. Jones CK, Schlosser MJ, van Zijl PC, Pomper MG, Golay $\mathrm{X}$, Zhou J. Amide proton transfer imaging of human brain tumors at 3T. Magn Reson Med 2006;56:585-92.

63. Zhou J, Hong X, Zhao X, Gao JH, Yuan J. APT-weighted and NOE-weighted image contrasts in glioma with different RF saturation powers based on magnetization transfer ratio asymmetry analyses. Magn Reson Med 2013;70:320-7.

64. Zhou J, Blakeley JO, Hua J, Kim M, Laterra J, Pomper MG, van Zijl PC. Practical data acquisition method for human brain tumor amide proton transfer (APT) imaging.
Magn Reson Med 2008;60:842-9.

65. Heo HY, Zhang Y, Jiang S, Lee DH, Zhou J. Quantitative assessment of amide proton transfer (APT) and nuclear overhauser enhancement (NOE) imaging with extrapolated semisolid magnetization transfer reference (EMR) signals: II. Comparison of three EMR models and application to human brain glioma at 3 Tesla. Magn Reson Med 2016;75:1630-9.

66. Jin T, Wang P, Zong X, Kim SG. MR imaging of the amide-proton transfer effect and the $\mathrm{pH}$-insensitive nuclear overhauser effect at 9.4 T. Magn Reson Med 2013;69:760-70.

67. Rerich E, Zaiss M, Korzowski A, Ladd ME, Bachert P. Relaxation-compensated CEST-MRI at $7 \mathrm{~T}$ for mapping of creatine content and $\mathrm{pH}$--preliminary application in human muscle tissue in vivo. NMR Biomed 2015;28:1402-12.

68. Shah SM, Mougin OE, Carradus AJ, Geades N, Dury R, Morley, W. and Gowland, P.A. The z-spectrum from human blood at 7T. Neuroimage 2018;167:31-40.

69. Heo HY, Zhang Y, Jiang S, Zhou J. Influences of experimental parameters on chemical exchange saturation transfer (CEST) metrics of brain tumors using animal models at 4.7T. Magn Reson Med 2019;81:316-30.

70. Zhao X, Wen Z, Huang F, Lu S, Wang X, Hu S, Zu D, Zhou J. Saturation power dependence of amide proton transfer image contrasts in human brain tumors and strokes at 3 T. Magn Reson Med 2011;66:1033-41.
Cite this article as: Zhang L, Zhao Y, Chen Y, Bie C, Liang Y, He X, Song X. Voxel-wise Optimization of Pseudo Voigt Profile (VOPVP) for Z-spectra fitting in chemical exchange saturation transfer (CEST) MRI. Quant Imaging Med Surg 2019;9(10):1714-1730. doi: 10.21037/qims.2019.10.01 

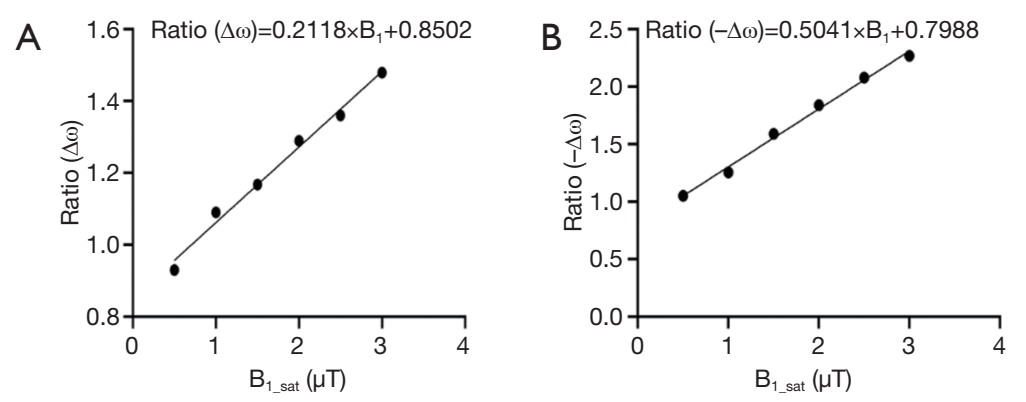

Figure S1 Relationship between (A) Ratio $(\Delta \omega)$, (B) Ratio $(-\Delta \omega)$, and $B_{1_{\text {sat }}}$ at $3 \mathrm{~T}$.

Table S1 Parameters for the Bloch Equation-Based simulations at 3T (5-pool) (48)

\begin{tabular}{lccccc}
\hline Parameters & Water & Amide $(3.5 \mathrm{ppm})$ & Amine $(2 \mathrm{ppm})$ & NOE $(-3.5 \mathrm{ppm})$ & MT $(0 \mathrm{ppm})$ \\
\hline $\mathrm{f}_{\mathrm{s}}$ & 1 & 0.004 & 0.002 & 0.0033 & 0.04 \\
$\mathrm{~K}_{\mathrm{sw}}\left(\mathrm{s}^{-1}\right)$ & - & 30 & 1000 & 20 & 20 \\
$\mathrm{~T}_{1}(\mathrm{~s})$ & 1.4 & 1.4 & 1.4 & 1.4 & 1.4 \\
$\mathrm{~T}_{2}(\mathrm{~ms})$ & 100 & 50 & 50 & 0.4 & $20 \times 10^{-3}$ \\
\hline
\end{tabular}

A

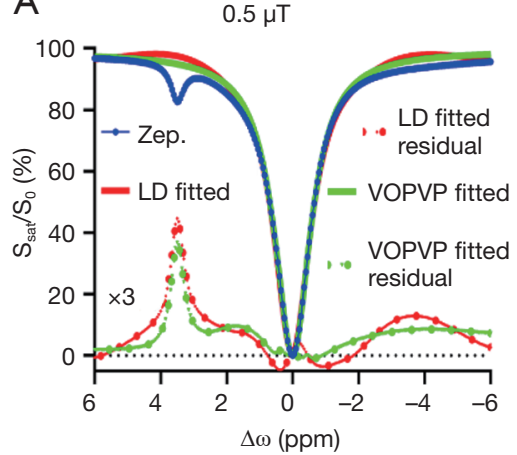

$\Delta \omega(\mathrm{ppm})$
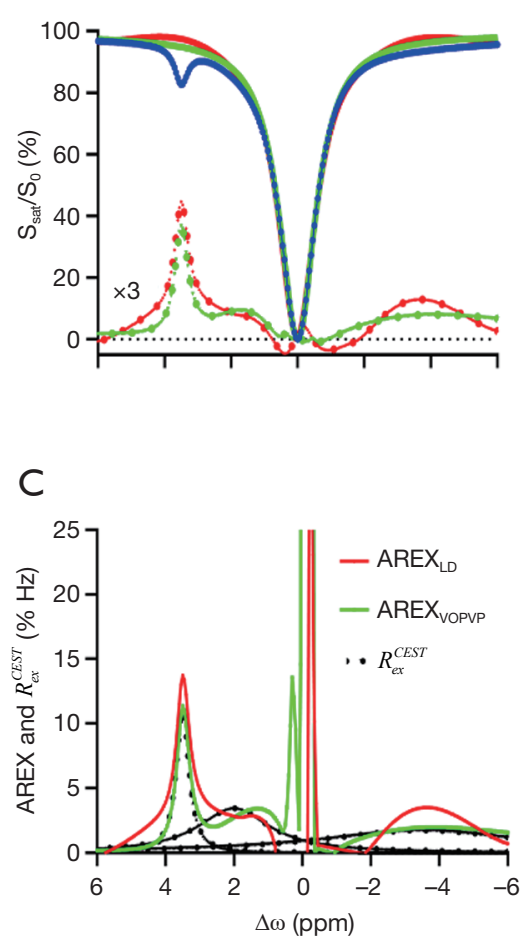

D

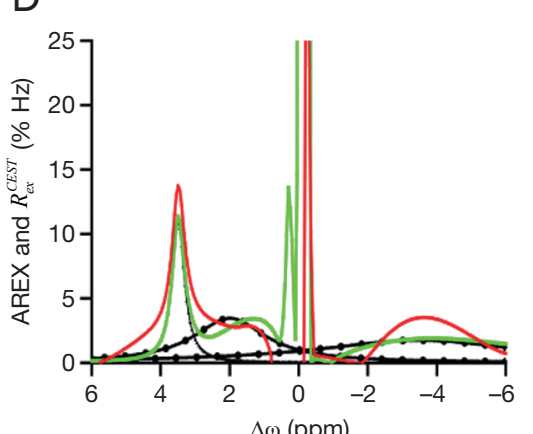

$1.0 \mu \mathrm{T}$

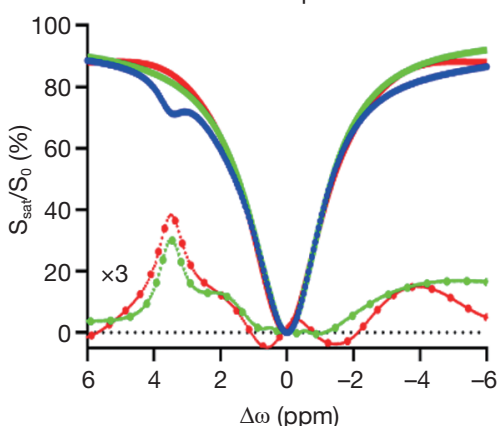

$\Delta \omega(\mathrm{ppm})$
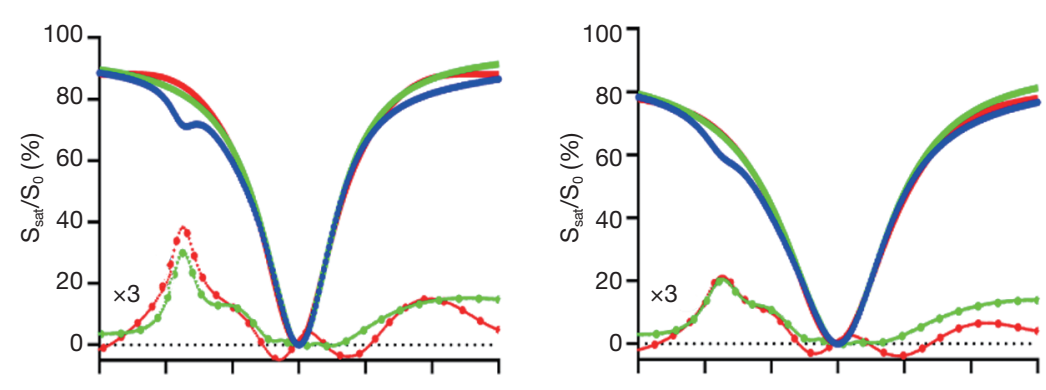

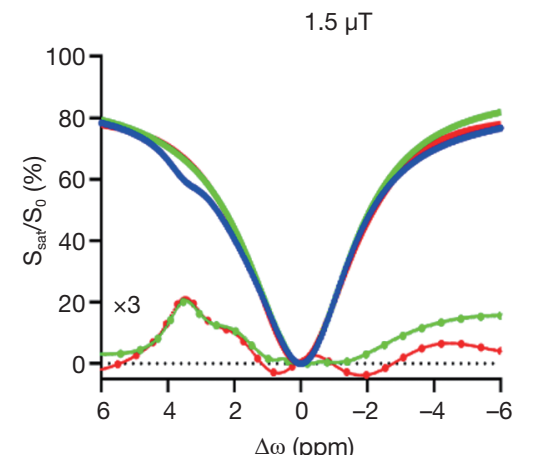

$\Delta \omega(\mathrm{ppm})$

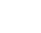

